\title{
Organoids from pituitary as a novel research model toward pituitary stem cell exploration
}

\author{
Benoit Cox ${ }^{1}$, Emma Laporte ${ }^{1}$, Annelies Vennekens ${ }^{1}$, Hiroto Kobayashi ${ }^{1,2}$, Charlotte Nys ${ }^{1}$, Indra Van Zundert ${ }^{3}$, \\ Hiroshi Uji-i³, Alizée Vercauteren Drubbel ${ }^{4}$, Benjamin Beck ${ }^{4,5}$, Heleen Roose', Matteo Boretto ${ }^{1}$ and \\ Hugo Vankelecom ${ }^{1}$
}

\begin{abstract}
'Department of Development and Regeneration, Cluster of Stem Cell and Developmental Biology, Unit of Stem Cell Research, KU Leuven (University of Leuven), Leuven, Belgium

2Department of Anatomy and Structural Science, Yamagata University Faculty of Medicine, Yamagata, Japan

${ }^{3}$ Department of Chemistry, Laboratory of Molecular Imaging and Photonics, KU Leuven, Leuven, Belgium

${ }_{4}^{4}$ nstitut de Recherche Interdisciplinaire en Biologie Humaine et Moléculaire (IRIBHM), Brussels, Belgium

5WELBIO, Université Libre de Bruxelles (ULB), Brussels, Belgium
\end{abstract}

Correspondence should be addressed to H Vankelecom: hugo.vankelecom@kuleuven.be

\begin{abstract}
The pituitary is the master endocrine gland, harboring stem cells of which the phenotype and role remain poorly characterized. Here, we established organoids from mouse pituitary with the aim to generate a novel research model to study pituitary stem cell biology. The organoids originated from the pituitary cells expressing the stem cell marker SOX2 were long-term expandable, displayed a stemness phenotype during expansive culture and showed specific hormonal differentiation ability, although limited, after subrenal transplantation. Application of the protocol to transgenically injured pituitary harboring an activated stem cell population, resulted in more numerous organoids. Intriguingly, these organoids presented with a cystic morphology, whereas the organoids from undamaged gland were predominantly dense and appeared more limited in expandability. Transcriptomic analysis revealed distinct epithelial phenotypes and showed that cystic organoids more resembled the pituitary phenotype, at least to an immature state, and displayed in vitro differentiation, although yet moderate. Organoid characterization further exposed facets of regulatory pathways of the putative stem cells of the pituitary and advanced new injury-activated markers. Taken together, we established a novel organoid research model revealing new insights into the identity and regulation of the putative pituitary stem cells. This organoid model may eventually lead to an interesting tool to decipher pituitary stem cell biology in both healthy and diseased gland.
\end{abstract}

\section{Introduction}

The pituitary represents the master endocrine gland, producing hormones that are key regulators of fundamental physiological processes including body growth, fertility, metabolism, immunity and stress. The gland produces these hormones through specialized endocrine cell types mainly present in its anterior lobe (anterior pituitary or AP). To achieve tight regulation of the physiological processes, the pituitary must control its hormonal output, and hence, also its endocrine cell composition in a strict way, both in basal and hormone-demanding conditions. 
A key player in achieving appropriate cell remodeling in the gland may be the local stem cells since tissue stem cells typically generate new functional cells during homeostatic turnover and dynamic adaptation processes. Since 2005, evidence has been accumulating that the pituitary gland, although indolent in turnover, contains a population of stem cells (Chen et al. 2005, 2009, Fauquier et al. 2008, Garcia-Lavandeira et al. 2009). Despite the identification of a series of markers with SOX2 at the apex, the molecular phenotype of these putative pituitary stem cells has not comprehensively been characterized. Even more, their functional role in the gland is far from understood. Recent lineage tracing studies provided support for a role in adult pituitary turnover with generation of endocrine cells, although occurring at a very low rate (Andoniadou et al. 2013, Rizzoti et al. 2013) which points to a strongly dormant character of the adult pituitary stem cells. Of note, major depletion of the SOX2+ (stem) cells does not affect hormonal cell homeostasis, thereby questioning a chief role in adult pituitary turnover (Roose et al. 2017). A more activated pituitary stem cell phenotype is observed during the early-postnatal growth and maturation phase of the gland. A higher proportion of cells showing boosted molecular and functional stemness character are present during the first weeks after birth (Gremeaux et al. 2012), and higher contribution to endocrine cells was found in lineage tracing following induction at neonatal age (Andoniadou et al. 2013, Rizzoti et al. 2013). Activation of the pituitary stem cell compartment has also been observed in the adult pituitary, more specifically following injury in the gland. Diphtheria toxin (DT)-induced ablation of growth hormone (GH)-producing cells in the GHCre/ iDTR (inducible DT receptor) mouse model is followed by a prompt proliferative and stemness pathway activation of the local stem cells (Fu et al. 2012, Willems et al. 2016). Moreover, several SOX2+ stem cells start to express GH which is likely underlying the $\mathrm{GH}^{+}$cell regeneration as observed during the following months. Taken all together, knowledge on pituitary stem cells is gradually increasing but stays fragmentary, and much remains to be uncovered regarding molecular characteristics and mechanisms underlying pituitary stem cell biology. Main obstacles to profoundly study pituitary stem cells are the scarcity and limitations of current in vitro research models. For example, stem cell-derived pituispheres generate only low amounts of study material (Chen et al. 2005, 2009, Fauquier et al. 2008, Garcia-Lavandeira et al. 2009). Moreover, the few pituitary cell lines available predominantly represent hormonal lineages, and do not faithfully reproduce primary pituitary tissue because of their tumor transformation and non-physiological 2D culture conditions.

Over the last decade, so-called organoids have been developed from manifold organs as powerful tools to study tissue (stem cell) biology (Clevers 2016). Organoids recapitulate many phenotypical and functional aspects of the original tissue's epithelium (Sato et al. 2009, Barker et al. 2010, Gao et al. 2014, Huch et al. 2015, Clevers 2016, Boretto et al. 2017). These 3D structures self-form in vitro from the tissue's stem cells which self-renew, expand and self-organize when embedded in an extracellular matrix scaffold (like Matrigel) and provided with the appropriate stem cell niche growth and regulatory factors. Typically, the Wingless-type MMTV integration site (WNT) pathway, further boosted by activation of leucinerich repeat-containing $G$ protein-coupled receptors (LGR) by R-spondin (RSPO) ligands, is implicated in organoid development. Further typical factors added to induce organoid formation from tissue stem cells are epidermal growth factor (EGF), the bone morphogenetic protein inhibitor Noggin and a transforming growth factor- $\beta$ (TGFB) inhibitor (Sato et al. 2009, Barker et al. 2010, Gao et al. 2014, Huch et al. 2015, Clevers 2016). Yet, each individual tissue requires further detailed medium optimization for organoid development and growth, based on the tissue's stem cell niche regulatory signals. At present, organoids have been established from manifold tissues (such as the intestine, liver, endometrium; Sato et al. 2011, Huch et al. 2015, Boretto et al. 2017), but not from (adult) pituitary. Recently, 3D structures have been developed from pluripotent embryonic stem cells (ESCs) that recapitulate pituitary development by applying embryogenetic principles (Suga et al. 2011, Ozone et al. 2016). Presently, organ-mimicking structures developing from ESC (or their somatic cell-derived counterparts, i.e. induced pluripotent stem cells or iPSCs) through the application of embryogenesis-reproducing pathways, are also termed 'organoids', but differ from the adult stem cellderived organoids in set-up and concept. No organoids have been reported yet starting from pituitary tissue.

Here, we aimed to establish organoids from adult mouse pituitary of both basal and activated (damaged) conditions. Characterization of the organoids obtained exposed new aspects of the putative pituitary stem cells. This novel organoid model may eventually allow to delve deeper into pituitary stem cell biology - at first regarding molecular phenotype and regulatory pathways and then regarding regenerative activation upon injury. (c) 2019 Society for Endocrinology Published by Bioscientifica Ltd. Printed in Great Britain 


\section{Materials and methods}

\section{Animals}

$\mathrm{GHCre}^{+/-}$mice (expressing Cre under control of the rat $G H$ promoter; Luque et al. 2007) were crossed with homozygous $\mathrm{iDTR}^{+/+}$mice (harboring the diphtheria toxin receptor (DTR) gene in the Rosa26 locus preceded by a floxed STOP cassette; Buch et al. 2005). The doubletransgenic $\mathrm{GHCre}^{+/-} ; \mathrm{iDTR}^{+/-}$offspring (hemizygous for both transgenes, further referred to as GHCre/iDTR) expresses DTR in GH-producing cells, thereby rendering these cells susceptible to DT-induced apoptosis (Fu et al. 2012, Willems et al. 2016). These double-transgenic mice were also crossed with Lgr5lacZ reporter mice (as obtained from Dr Clevers, Hubrecht Institute, Utrecht, The Netherlands; Barker et al. 2007). Sox2eGFP mice, expressing enhanced GFP in SOX2+ cells (Ellis et al. 2004), were used to sort SOX2 $2^{\text {eGFPpos }}$ and SOX2 $2^{\text {eGFPneg }}$ cells. To obtain Sox2CreERT2+/-;eYFPfl/- double-transgenic mice, Sox2CreERT2+/- mice (obtained from Dr Martinez-Barbera, UCL, London; Andoniadou et al. 2013) were crossed with eYFPfl/fl reporter mice (harboring the enhanced yellow fluorescent protein/eYFP gene in the Rosa26 locus preceded by a floxed STOP cassette; obtained from Dr. Carmeliet, KU Leuven). NOD-SCID mice were provided by in-house breeding. Genotyping of all mouse models was performed as previously described (Barker et al. 2007, Fu et al. 2012, Roose et al. 2017). Mice were bred on a C57BL/6 background and kept at the university's animal housing facility (KU Leuven, Belgium), providing constant temperature, humidity, day/night cycle and water and food ad libitum. Animal experiments were approved by the KU Leuven Ethical Committee.

\section{Treatment of mice}

Adult (8-12 weeks old) male GHCre/iDTR mice and Crenegative control littermates (referred to as -/iDTR) were injected intra-peritoneally (i.p.) with DT $(4 \mathrm{ng} / \mathrm{g}$ body weight, twice a day; List Biological Laboratories, Campbell, CA, USA) for three consecutive days as described before (Fu et al. 2012, Willems et al. 2016). Mice were sacrificed at day 11 following start of DT treatment (day of first injection = day 1 ).

Sox2CreERT2+/-;eYFPfl/- mice were injected i.p. with tamoxifen (Sigma-Aldrich; dissolved in corn oil; $0.15 \mathrm{mg} / \mathrm{g}$ body weight) for two consecutive days and were killed 2 days after the last injection.

\section{Organoid culture}

The pituitary was isolated from killed mice, and the AP was separated and dispersed into single cells using trypsin as previously described (Chen et al. 2005). AP cells (10,000 unsorted cells or 8000 FACS-sorted cells, see below) were embedded in a $30 \mu \mathrm{L}$ drop composed of serum-free defined medium (SFDM; Thermo Fisher Scientific) and Growth Factor Reduced Matrigel (Corning) in a 30:70 ratio and seeded in 48-well plates (Corning) (Supplementary Fig. 1A, see section on supplementary data given at the end of this article). The jellified droplets were cultured in SFDM containing a cocktail of growth and regulatory factors. For all cultures, 'pituitary organoid medium' (PitOM, Table 1) was used unless otherwise stated. At seeding of the primary cells or replating of the organoidderived fragments or cells (i.e. passaging), ROCK inhibitor (Y27632, 10 $\mathrm{M}$; Merck, Overijse, Belgium) was added to the medium. Cultures were kept at $37^{\circ} \mathrm{C}$ in a $1.9 \% \mathrm{CO}_{2}$ incubator and medium was refreshed every 2-3 days. For quantification, all organoid structures with a diameter $\geq 50 \mu \mathrm{m}$ were counted. In a few experiments, AP cells were first cultured in free-floating sphere-forming conditions as previously described (Chen et al. 2005, 2009, Roose et al. 2017). After 6 days, the formed pituispheres were embedded in Matrigel for organoid culture as described above.

Expanding organoid cultures were passaged every 7-10 days (Supplementary Fig. 1A). Organoids were collected and incubated with TrypLE Express (Thermo Fisher Scientific) for $5 \mathrm{~min}$ (cystic organoids) or $10 \mathrm{~min}$ (dense organoids) at $37^{\circ} \mathrm{C}$. After TrypLE inactivation, the suspension was centrifuged at $200 \boldsymbol{g}$, the pellet was resuspended in SFDM and the organoids were dispersed by vigorous pipetting yielding mostly single cells (dense organoids) or cells and organoid fragments (cystic organoids). After centrifugation, the pellet was resuspended in 70\% Matrigel, replated and cultured as described earlier. AP cells from Sox $2 \mathrm{eGFP}^{+/-}$adult mice were sorted by FACS (Aria III, BD Biosciences, Erembodegem, Belgium) into SOX2 ${ }^{\text {eGFPpos }}$ and SOX2 $2^{\text {eGFPneg, }}$ cells which were used for organoid culturing or RNA isolation (see below). Brightfield and fluorescence pictures of organoid cultures were recorded using the Axiovert 40 CFL microscope (Zeiss). To assess WNT pathway involvement, IWP2 $(2 \mu \mathrm{M}$; Sigma-Aldrich) and XAV939 (10 $\mathrm{M}$; Sigma-Aldrich) were added to the medium. Heparin-binding epidermal growth factor-like growth factor (HB-EGF; Peprotech, Rocky Hill, NJ, USA) and TGFB1 (R\&D systems) were added at 
Table 1 Composition of the pituitary organoid medium (PitOM).

\begin{tabular}{l}
\hline Componenta \\
\hline Serum-free defined medium (SFDM) \\
B27 (without vitamin A) \\
N2 \\
L-Glutamine \\
N-acetyl-L-cysteine \\
Nicotinamide \\
EGF \\
A83-01 (activin receptor-like kinase (Alk) 4/5/7 inhibitor) \\
SB202190 (p38 mitogen-activated protein kinase inhibitor) \\
IGF1 \\
Recombinant human (rh) WNT3A* \\
rhRSPO1* \\
FGF2 (= basic FGF)* \\
FGF8* \\
FGF10* \\
SHH* \\
Noggin* \\
Cholera toxin*
\end{tabular}

\begin{tabular}{l}
\hline Concentration \\
\hline $2 \%$ \\
$1 \%$ \\
$2 \mathrm{mM}$ \\
$1.25 \mathrm{mM}$ \\
$10 \mathrm{mM}$ \\
$50 \mathrm{ng} / \mathrm{mL}$ \\
$0.5 \mu \mathrm{M}$ \\
$10 \mu \mathrm{M}$ \\
$100 \mathrm{ng} / \mathrm{mL}$ \\
$200 \mathrm{ng} / \mathrm{mL}$ \\
$200 \mathrm{ng} / \mathrm{mL}$ \\
$20 \mathrm{ng} / \mathrm{mL}$ \\
$200 \mathrm{ng} / \mathrm{mL}$ \\
$100 \mathrm{ng} / \mathrm{mL}$ \\
$100 \mathrm{ng} / \mathrm{mL}$ \\
$100 \mathrm{ng} / \mathrm{mL}$ \\
$100 \mathrm{ng} / \mathrm{mL}$
\end{tabular}

\begin{tabular}{l}
\hline Supplier \\
\hline Thermo Fisher Scientific \\
Thermo Fisher Scientific \\
Thermo Fisher Scientific \\
Thermo Fisher Scientific \\
Sigma-Aldrich \\
Sigma-Aldrich \\
R\&D Systems \\
Sigma-Aldrich \\
Sigma-Aldrich \\
PeproTech \\
R\&D Systems \\
R\&D Systems \\
R\&D Systems \\
PeproTech \\
PeproTech \\
R\&D Systems \\
R\&D Systems \\
Sigma-Aldrich
\end{tabular}

aComponents marked with * are absent in EASI medium.

a concentration of $10 \mathrm{ng} / \mathrm{mL}$ and $5 \mathrm{ng} / \mathrm{mL}$, respectively. GHCre/iDTR AP-derived (cystic) organoids were treated with $10 \mathrm{ng} / \mathrm{mL}$ DT or vehicle (PBS) in PitOM for $24 \mathrm{~h}$. This in vitro DT treatment has before been shown to induce $\mathrm{GH}^{+}$cell ablation in GHCre/iDTR AP cell cultures and aggregates (Luque et al. 2011 and data not shown). Unless otherwise stated, organoids of passage (P) 2-4 were used for the analyses described below. Of note, pituitary organoids could also be obtained from mouse strains other than C57BL/6 such as FVB and CD1 (data not shown).

\section{Organoid differentiation}

To test the differentiation capacity of pituitary organoids in vitro, organoids were passaged and first grown in organoid culture medium for 1 week. Then, organoids were replated (without dissociation) in Matrigel drops and cultured in SFDM (without growth factors) or SFDM + retinoic acid (RA, $1 \mu \mathrm{M}$; Sigma-Aldrich) for 4 days followed by SFDM+RA+dexamethasone (Dex, 50nM; Sigma-Aldrich) for 2 days, a protocol previously reported to induce $\mathrm{GH}^{+}$cell differentiation (Cheng et al. 2011). In an additional approach, formed organoids were cultured for 7 days in SFDM on Matrigel-coated coverslips as previously described for pituispheres (Chen et al. 2005, 2009, Roose et al. 2017).

To test the differentiation capacity in vivo, pituitary $\mathrm{eYFP}^{+}$organoids (more specifically, dense organoids expanded from tamoxifen-injected Sox2CreERT2 ${ }^{+/-}$;eYFPfl/- mice) or PBS (sham) were injected under the kidney capsule of 8- to 10-week-old male
NOD-SCID mice. After 2, 4 or 6 weeks, mice were killed and the grafted kidney was dissected. The kidneys were photographed using the Discovery V12 stereo microscope (Zeiss) and were further processed for immunofluorescent analysis (see below) of SOX2 or the pituitary hormones $\mathrm{GH}$, prolactin (PRL), adrenocorticotropic hormone $(\mathrm{ACTH})$ and glycoprotein hormone $\alpha$-subunit (AGSU; i.e. the common subunit of thyroid-stimulating hormone (TSH), luteinizing hormone (LH) and follicle-stimulating hormone (FSH)).

\section{Histochemical analysis and immunostaining}

Tissues and organoids were fixed in 4\% PFA at room temperature for $3 \mathrm{~h}$ and $1 \mathrm{~h}$, respectively, and embedded in paraffin using the Excelsior ES Tissue Processor (Thermo Fisher Scientific). Sections were subjected to hematoxylin and eosin (H\&E) staining, RNA in situ hybridization or immunofluorescence/immunohistochemical staining. For analysis of the in vitro differentiation experiments, organoids were either analyzed as a whole or dispersed and dissociated cells spun onto cytospin slides (Roose et al. 2017). Immunostainings were performed after antigen retrieval with $10 \mathrm{mM}$ citrate buffer $(\mathrm{pH} \mathrm{6)}$ and permeabilization with Triton X-100(0.1\% inPBS). Following incubation with primary and secondary antibodies (Supplementary Table 1), sections were covered with Vectashield (containing 4',6-diamidino-2-phenylindole or DAPI; Vector Laboratories, Peterborough, UK) or DPX mountant (Sigma-Aldrich) after counterstaining with hematoxylin. RNA in situ hybridization was performed 
using the RNAscope 2.0 Fast Red Detection Kit (Advanced Cell Diagnostics, Newark, CA, USA), according to the manufacturer's protocol. Immunofluorescence staining of SOX9 was done in $7 \mu \mathrm{m}$ cryosections of organoids obtained using a CM1860 Leica cryostat (Leica Biosystems) after 20-min fixation with 4\% PFA and embedding in optimal cutting temperature compound (OCT; Sakura, Torrance, CA, USA). Processed sections were analyzed with a Leica DM5500 epifluorescence microscope (Leica Microsystems) accessible through InfraMouse (KU Leuven-VIB) or an Imager.Z2 microscope and Zen software (Zeiss). Organoids cultured on coverslips were analyzed using a Zeiss LSM 880 confocal microscope. Recorded images were converted into pictures for figures with ImageJ (http://imagej.nih.gov/ij/).

\section{Two-photon excitation microscopy}

Organoids were fixed with $4 \%$ PFA for $30 \mathrm{~min}$ at $37^{\circ} \mathrm{C}$ and incubated with Hoechst33342 $(2.5 \mu \mathrm{g} / \mathrm{mL}$ in PBS; Sigma-Aldrich) for $1 \mathrm{~h}$ at $37^{\circ} \mathrm{C}$. Then, organoids and cell membranes were permeabilized with $0.1 \%$ Triton X-100 (in 1\% bovine serum albumin/BSA in PBS). After blocking with 1\% BSA/PBS, organoids were incubated with Phalloidin-Atto520 (Sigma-Aldrich) for $40 \mathrm{~min}$ at room temperature. Z-stacks (up to $350 \mu \mathrm{m}$ ) of the organoids were acquired with a confocal microscope (Leica TCS SP8 X; Leica Microsystems) equipped with a Mai Tai DeepSee multiphoton laser (Spectra Physics, Vienna, Austria) and a 20×/0.75 water immersion objective (HC PL APO CS2; Leica Microsystems). Hoechst33342 was excited at $700 \mathrm{~nm}$ and Phalloidin-Atto520 at $1040 \mathrm{~nm}$ by two-photon excitation, and emission detected using hybrid detectors and refractive light detectors (Leica Microsystems) set at the appropriate detection window.

\section{X-gal staining}

Organoids and pituitaries were fixed for $15 \mathrm{~min}$ at room temperature in a mixture of $5 \mathrm{mM}$ ethylene glycol tetraacetic acid (EGTA), $2 \mathrm{mM} \mathrm{MgCl}_{2}$ and $0.2 \%$ glutaraldehyde in PBS. Samples were rinsed twice for 5 min with PBS containing $2 \mathrm{mM} \mathrm{MgCl}_{2}, 0.01 \%$ sodium deoxycholate and $0.02 \% \mathrm{NP}-40$. Then, samples were covered and incubated overnight (pituitaries) or for $2 \mathrm{~h}$ (organoids), at $37^{\circ} \mathrm{C}$ with $\mathrm{X}$-gal staining solution $\left(1 \mathrm{mg} / \mathrm{mL}\right.$ X-gal, $2 \mathrm{mM} \mathrm{MgCl}_{2}, 5 \mathrm{mM} \mathrm{K}_{3} \mathrm{Fe}(\mathrm{CN})_{6}, 5 \mathrm{mM}$ $\mathrm{K}_{4} \mathrm{Fe}(\mathrm{CN})_{6}, \quad 0.01 \%$ sodium deoxycholate and $0.02 \%$ NP-40 in PBS; all reagents from Sigma-Aldrich). After incubation, pituitaries were rinsed three times for $10 \mathrm{~min}$, post-fixed overnight at $4^{\circ} \mathrm{C}$ and processed for paraffin embedding. Sections were counterstained with Eosin $\mathrm{Y}$ (Sigma-Aldrich).

\section{Transmission electron microscopy}

Organoids were fixed overnight in 2.5\% glutaraldehyde (Grade I, Sigma-Aldrich) in $0.1 \mathrm{M}$ sodium cacodylate buffer $(\mathrm{pH} 7.2)$ at $4^{\circ} \mathrm{C}$. The fixed organoids were pelleted at $200 \mathrm{~g}$ and washed three times in $0.1 \mathrm{M}$ cacodylate buffer. The pellets were resuspended in $2.0 \%$ low melting point agarose (Thermo Fisher Scientific) and centrifuged at $1000 \mathrm{~g}$. After solidification, the pellets were cut up in small cubes, washed and post-fixed in 1\% osmium tetroxide and $1.5 \%$ potassium ferrocyanide in $0.1 \mathrm{M}$ cacodylate buffer, washed again and mordanted by $0.1 \%$ tannic acid for $20 \mathrm{~min}$. Then, samples were stained overnight en bloc with uranyl acetate (Agar Scientific, Stansted, UK) followed by 30-min lead aspartate incubation with intermittent washing in cacodylate buffer, dehydrated in a graded ethanol series and propylene oxide and finally embedded in epoxy resin (Agar 100; Agar Scientific). The embedded samples were polymerized for 2 days at $60^{\circ} \mathrm{C}$. Thin $70 \mathrm{~nm}$ sections were cut with a UCT ultramicrotome (Leica) and analyzed using the JEM1400 transmission electron microscope (JEOL, Zaventem, Belgium) equipped with an Olympus SIS Quemesa 11Mpxl camera.

\section{Gene expression analysis by RT-qPCR}

Total RNA was extracted from organoids or dissociated AP cells with the RNeasy Micro kit (Qiagen) and subjected to reverse transcription (RT) using the Superscript III FirstStrand Synthesis Supermix (Thermo Fisher Scientific), all according to the manufacturers' recommendations. Resultant cDNA samples were subjected to SYBR green-based quantitative real-time PCR (qPCR) using the StepOnePlus Real-Time PCR System (AB Applied Biosystems). All qPCR reactions were performed with Platinum SYBR Green qPCR SuperMix-UDG mastermix (Thermo Fisher Scientific). Forward and reverse primers (IDT Technologies, Haasrode, Belgium; Supplementary Table 2) were designed using PrimerBank (Spandidos et al. 2010) and PrimerBLAST (https://www.ncbi. nlm.nih.gov/tools/primer-blast/). Hypoxanthine phosphoribosyltransferase (Hprt) and $\beta$-actin (Actb) were tested and used as housekeeping genes. Relative gene expression levels were calculated as $\Delta \mathrm{Ct}$ values (Ct 'target' minus Ct 'average housekeeping genes') and expressed as fold change $\left(2^{-(\Delta \Delta \mathrm{Ct})}\right)$ in comparison to a reference as 
specified in the figures. For some of the experiments, amplicons were visualized using gel electrophoresis in a $2 \%$ agarose gel with SYBR Safe (Thermo Fischer Scientific).

\section{RNA-sequencing analysis}

RNA extracted from organoids or FACS-sorted (SOX2 ${ }^{\text {eGFPpos})}$ AP cells was checked for RNA Integrity Number (RIN) using Agilent Picochips on an Agilent BioAnalyzer 2100 (Agilent Technologies). Using good-quality samples (RIN $>7.5$ ), RNA-seq was performed in collaboration with the Nucleomics Core (VIB/KU Leuven). First, RNA samples were amplified using Smart-Seq v4 kit (Takara Bio Europe, St-Germain-en-Laye, France). RNA library preparation was executed with the NEBNext Ultra DNA Library prep kit, followed by sequencing on a NextSeq 500 instrument (both from Illumina). Raw reads were subjected to quality control. Sequence reads were pre-processed to remove adaptors and filter low-quality reads. The high-quality reads were aligned to the mouse reference genome using Tophat (v2.0.13). Statistic modeling was performed using the DESeq 1.32.0 package of Bioconductor (http://www. bioconductor.org). Sequencing data are available from the NCBI Gene Expression Omnibus (GEO, http://www.ncbi. nlm.nih.gov/geo/) through accession number GSE116654. Gene ontology (GO) analysis of differentially expressed genes (defined by a ratio $\geq 5$ or $\leq 0.2$ and normalized counts $\geq 10$ ) was done using the Database for Annotation, Visualization and Integrated Discovery (DAVID, version 6.8; https://david.ncifcrf.gov/). Hierarchical clustering of selected gene lists was performed using Multiple experiment Viewer software, version 4.9.0 (http://mev.tm4.org/).

\section{Statistical analysis}

Statistical analysis was performed (for $n \geq 3$ ) using GraphPad Prism (version 5.01) and Microsoft Excel software (2016). Unpaired two-tailed $t$-Student test was applied for comparison of two groups. In case of multiple comparisons (more than two groups), one-way analysis of variance was done followed by Dunnett test. Statistical significance was defined as $P<0.05$.

\section{Results}

\section{Development of organoids from mouse pituitary}

To achieve the development of organoids from pituitary, we embedded dissociated cells from adult mouse AP in a 3D Matrigel scaffold and added culture medium containing the generic organoid culture components EGF, Noggin, WNT3A and RSPO1 (Supplementary Fig. 1A). In addition, we included components from pathways known to play a role in pituitary development and/or stem cell maintenance and proliferation, such as fibroblast growth factors (FGF), insulin-like growth factor-1 (IGF1) and sonic hedgehog (SHH) (Boretto et al. 2017, Cox et al. 2017). By testing these components in a trial-and-error manner, we eventually defined a culture medium (further referred to as 'pituitary organoid medium', PitOM; Table 1) in which organoid structures were found to develop, typically in a time span of 2 weeks (passage 0 , P0; Fig. 1A). Time-lapse recording supported that organoids developed from individual cells (Fig. 1A). Remarkably, two discernibly different organoid morphologies were obtained, i.e. dense (solid) organoids and cystic (hollow) organoids (i.e. with a lumen) (Fig. 1A). The independent cultures ( $n=14$ biological replicates) consisted either of only dense organoids (15\% of the cultures), cystic organoids (30\%) or a mixture of both $(55 \%$ of the cultures with a higher number of cystic vs dense organoids per culture) (Supplementary Fig. 1B and C). After 2 weeks, the organoids were dissociated into single cells and re-seeded (Supplementary Fig. 1A). Organoids formed again, but only the dense organoids could be efficiently passaged (Fig. 1B and Supplementary Fig. 1B). The cystic organoid cultures were not expandable and the cystic organoids in the mixed cultures quickly disappeared; consequently, only dense organoid cultures were retained (Supplementary Fig. 1B). At present, the dense organoids could be expanded up to P41 (i.e. 11 months of culture) (Fig. 1B).

\section{Biological characterization of the pituitary organoids}

Organoids typically originate from the stem cells of a tissue. The large majority of the pituitary organoid cells ( $\geq 85 \%$ ) were found to express the pituitary stem cell marker SOX2 (Fig. 1C). These SOX2+ cells co-expressed E-cadherin, which in the pituitary is also present in the SOX2+ cells (Fauquier et al. 2008, Garcia-Lavandeira et al. 2009, Roose et al. 2017). The SOX2-negative cells were mainly located in the inner region of the organoids and did not co-express E-cadherin (Fig. 1C; arrowheads).

To investigate whether the organoids are derived from the SOX2-expressing pituitary cells, we isolated SOX2 ${ }^{\text {eGFPpos }}$ cells by FACS from the AP of SOX2 reporter (Sox2eGFP) mice. Organoids developed in these cultures (starting from individual cells), while no organoids 


\begin{tabular}{l|l|l|l|l|}
\hline $\begin{array}{l}\text { Journal of } \\
\text { Endocrinology }\end{array}$ & B Cox et al. & Pituitary organoids & $240: 2$ & 293 \\
\hline
\end{tabular}

A
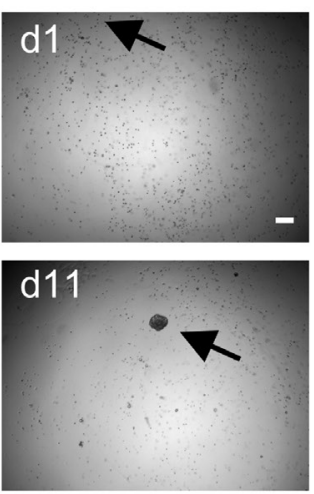

B

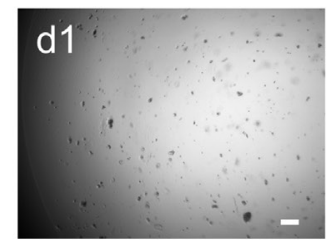

Organoid initiation (P0)
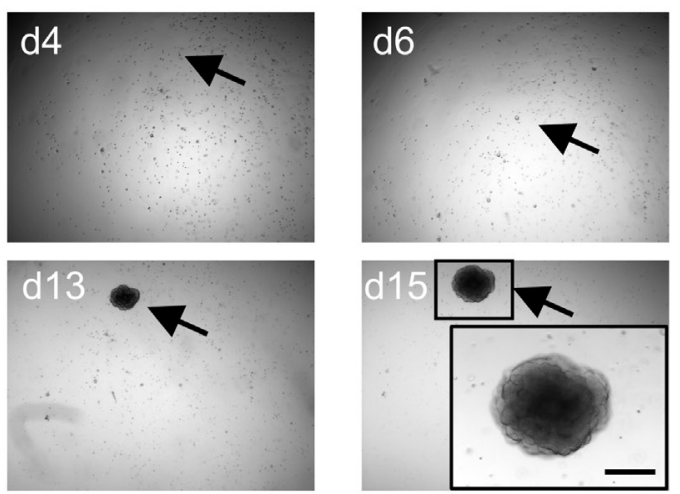

Organoid expansion

P3

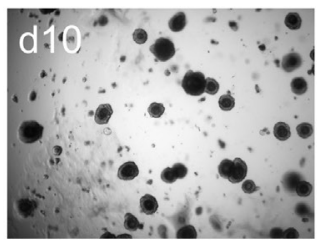

P10

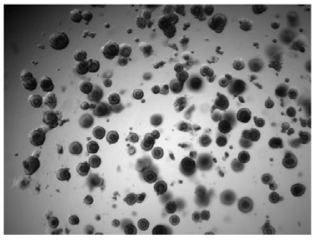

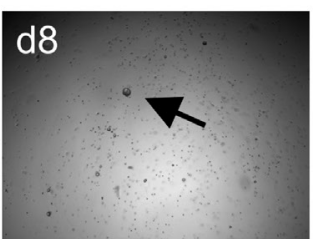

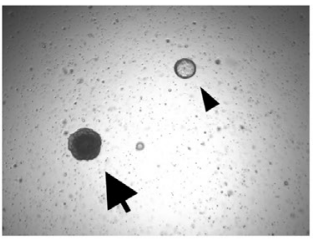

P41

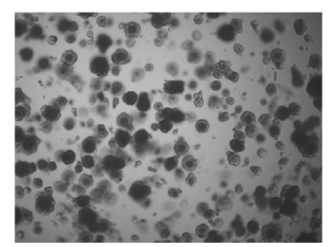

C
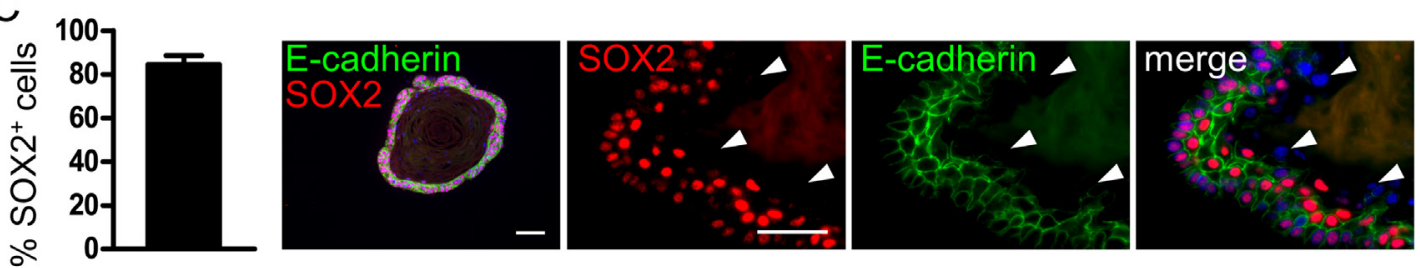

D Organoid initiation (P0)
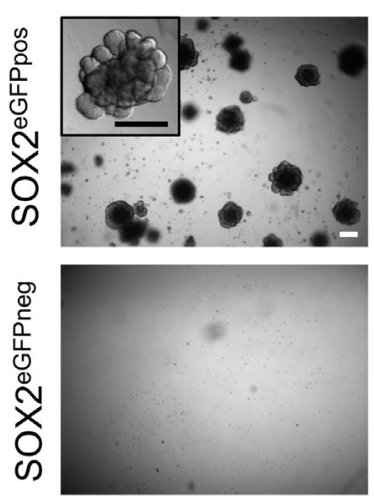
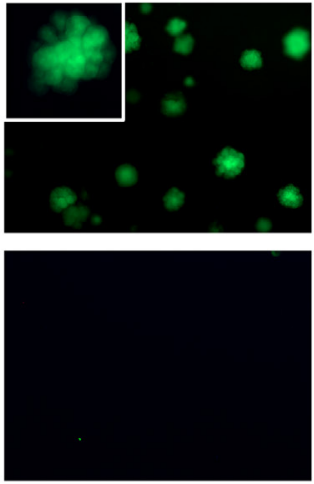

\section{E Organoid expansion (P2)}
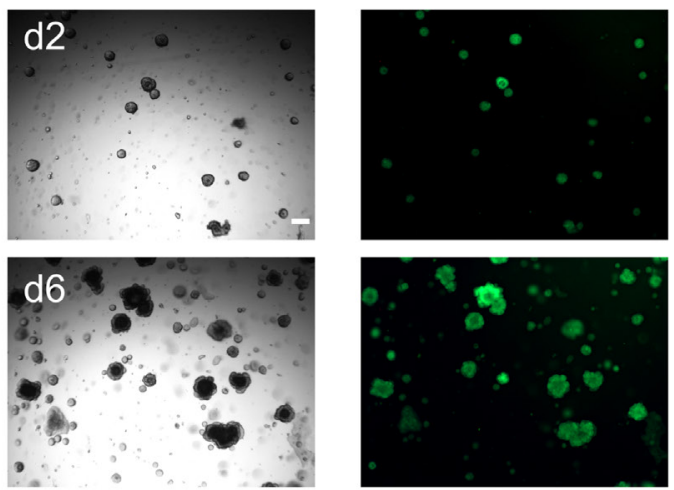

\section{Figure 1}

Development of SOX2+ cell-derived organoids from pituitary. (A) Formation of organoids starting from dissociated AP cells (passage 0 , P0). Representative light microscopic pictures are shown at different time points (seeding at day 1, d1), with the arrow pointing to the same structure at the consecutive days of culture, starting from a single cell. Inset: magnification of boxed area. Lower right panel: example of a culture at d13 containing both dense (arrow) and cystic (arrowhead) organoids. Scale bar: $200 \mu \mathrm{m}$. (B) Passaging and expansion of pituitary organoids, shown at P3 (meaning about 1 month in culture; pictures at 1 day/d1 and 10 days/d10 after seeding), P10 (3 months) and P41 (11 months). Organoids reform at passaging in 7-10 days. Scale bar: 200 um. (C) Percentage of SOX2+ cells in (dense) organoids (bar represents mean \pm S.E.M.; $n=3$ ) and double immunofluorescence staining of SOX2 (red) and E-cadherin (green) in paraffin-embedded organoid sections (composite overview and single-color magnified views). Arrowheads indicate SOX2-/Ecadherin- cells. Nuclei are stained with DAPI (blue). Scale bar: $50 \mu \mathrm{m}$. (D) AP SOX2eGFPos and SOX2eGFPneg cells from transgenic Sox2eGFP mice cultured in organoid-forming conditions (P0). Representative pictures are shown, including brightfield (left) and fluorescence image for eGFP expression (right). Scale bar: $200 \mu \mathrm{m}$. (E) Brightfield (left) and eGFP fluorescence images of SOX2eGFPpos organoids at P2 on d2 and d6 after replating. Scale bar: $200 \mu$ m. 
formed in the SOX2 $2^{\text {GFPneg }}$ cell cultures (Fig. 1D and Supplementary Fig. 1D). Moreover, a clear enrichment in organoid-forming cells was obtained in the SOX2 2 GFPpos cell fraction (Supplementary Fig. 1E). Of note, only a small number of the seeded SOX2 $2^{\text {eGFPpos }}$ cells gave rise to organoids (i.e. 0.14\%; Supplementary Fig. 1E), which is in agreement with previous studies in which SOX2+ stem cell activity was measured by pituisphere and colony formation capacity (Fauquier et al. 2008, Chen et al. 2009, Gaston-Massuet et al. 2011) (see 'Discussion' section). The organoids retained the SOX2 $2^{\text {eGFPpos }}$ phenotype during expansion (as assessed until P9 or 3 months in culture; Fig. 1E and data not shown). To investigate whether at passaging organoids also develop from individual cells and not by cell aggregation, we mixed organoid-derived fluorescent $(\mathrm{eYFP}+)$ cells (see below) with non-fluorescent (WT) cells. Only entirely fluorescent or non-fluorescent organoids were obtained (Supplementary Fig. 1F and G), thereby providing strong support for a clonal origin of the organoids.

To explore whether the pituitary organoids obtained could give rise to the gland's differentiated (hormonal) cells, several in vitro approaches were tested that may induce pituitary hormonal cell differentiation, including the addition of FBS (Garcia-Lavandeira et al. 2009), culture on Matrigel-coated surface (as effective for pituispheres; Fauquier et al. 2008, Chen et al. 2009) and treatment with retinoic acid (RA) and dexamethasone (Cheng et al. 2011). However, hormone expression was not detected after applying these protocols (Supplementary Fig. 2A and data not shown). Omission of all (stem cell) growth factors from the organoid medium did also not result in hormonal differentiation (Supplementary Fig. 2A and data not shown). It has been found that for certain tissues like the pancreas, the stem cell organoids have to be exposed to an in vivo environment to achieve differentiation (Huch et al. 2013a). Therefore, we transplanted expanded (dense) organoid cultures under the kidney capsule of NOD/SCID mice. To track the organoid cells, we developed eYFP+ organoids from a SOX2+ lineage tracing mouse model (i.e. Sox2CreERT2+/-;eYFPfl-; see 'Materials and methods' section) displaying expression of eYFP in the SOX2+ cells which remains expressed in the descendant cells (Fig. 2A). The eYFP+ organoids (not expressing hormones; data not shown) were subrenally grafted and analyzed 2, 4 and 6 weeks later (Fig. 2A). An eYFP+ graft could be clearly detected at all time points (Fig. 2B). After 2 weeks, a few PRL-immunopositive cells were observed while no GH, ACTH or AGSU (the common subunit of TSH, LH and FSH) could be detected in the graft (Fig. 2C and
Supplementary Fig. 2B). After 4 weeks, various cells were immunopositive for PRL, GH and AGSU, whereas after 6 weeks, no hormone immunoreactivity was detected anymore (Fig. 2C and Supplementary Fig. 2B). Thus, differentiation to hormone-expressing cells occurred in vivo although at limited degree. Many cells of the graft still expressed SOX2 at the time points analyzed (Fig. 2C).

\section{Different organoid phenotypes from normal and damaged pituitary}

Recently, we reported that the pituitary stem cells are activated upon injury in the gland as inflicted by DT-mediated $\mathrm{GH}^{+}$cell ablation using the GHCre/iDTR mouse model (Fu et al. 2012, Willems et al. 2016). Hence, we here addressed the question whether organoid culturing from damaged and normal (undamaged) pituitary would show differences. AP cells from DT-treated GHCre/iDTR and control (-/iDTR) mice were seeded in the organoidforming culture conditions as established above. A higher number of organoid structures were formed in the cell cultures from injured GHCre/iDTR pituitary than from undamaged -/iDTR gland (Fig. 3A and B). Intriguingly, the majority $(80 \%)$ of the cultures $(n=13$ biological replicates) of the damaged pituitary were exclusively composed of cystic organoids while the remaining part contained both cystic and dense organoids, with the cystic organoids present in large excess (Supplementary Fig. 3A and B). Time-lapse recording also supported a clonal origin of these cystic organoids (Supplementary Fig. 3C). The more abundant organoid formation from damaged (GHCre/iDTR) vs normal pituitary (Fig. 3A and B) was not due to the possible presence of DTR (alias HB-EGF) in the medium as could have been shed from ablated, disintegrating $\mathrm{GH}^{+}$cells. Although exogenously added HB-EGF could compensate for EGF in whose absence no organoid growth occurred, endogenous HB-EGF levels - if present - were not sufficient (Supplementary Fig. 3D). Moreover, adding HB-EGF to PitOM did not affect the abundance of cystic organoids (neither of dense organoids) (Supplementary Fig. 3E). It should further be noted that the AP was collected for organoid culture initiation 1 week after stopping the DT injection which makes it unlikely that large amounts of HB-EGF were still present in the organoid-forming AP cell cultures since the $\mathrm{GH}^{+}$cell ablation process occurs promptly upon DT treatment (from the first day) and is finalized 3-4 days later (Fu et al. 2012).

The organoids from the injured GHCre/iDTR AP could be passaged as well as the organoids from the control 


\begin{tabular}{|l|l|l|r|r|}
\hline Journal of & B Cox et al. & Pituitary organoids & $240: 2$ & 295 \\
Endocrinology & & & \\
\hline
\end{tabular}

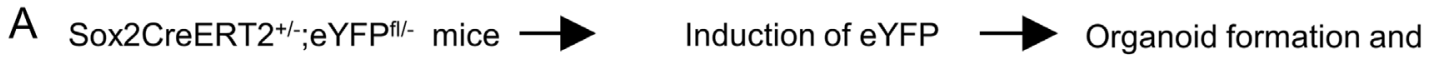
expression expansion

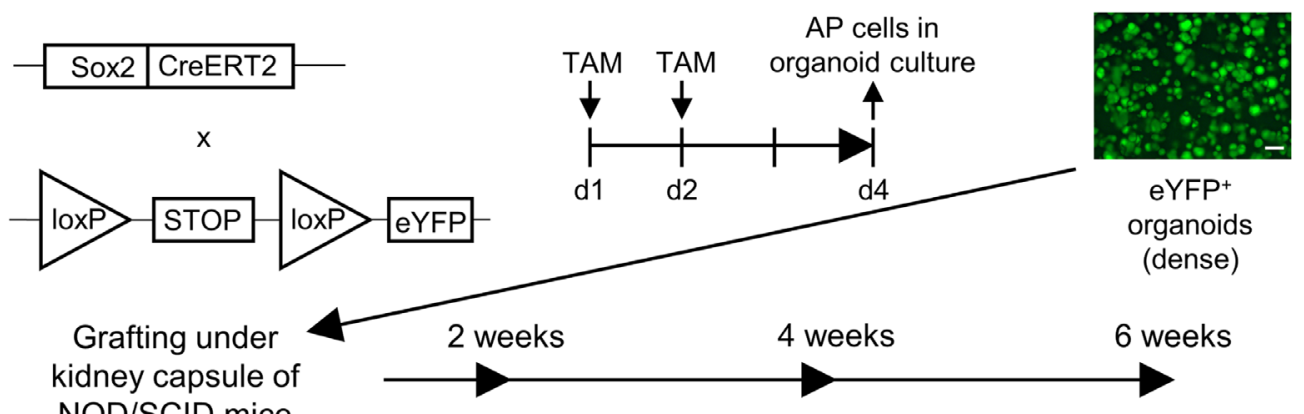

B

sham
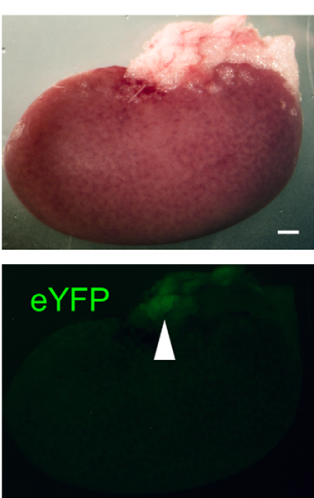

C
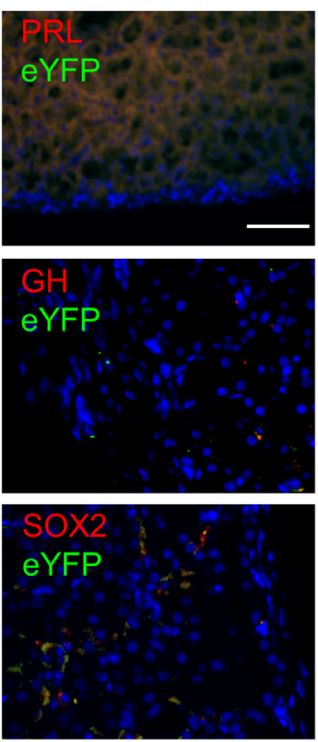

2 weeks
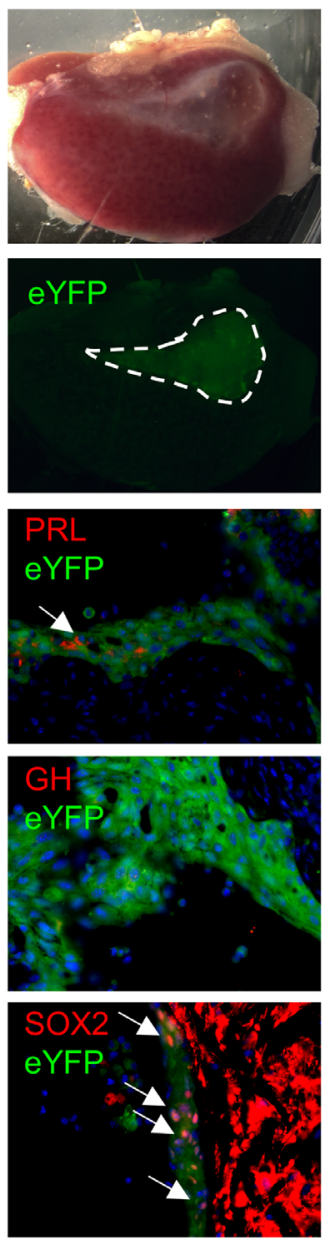

4 weeks
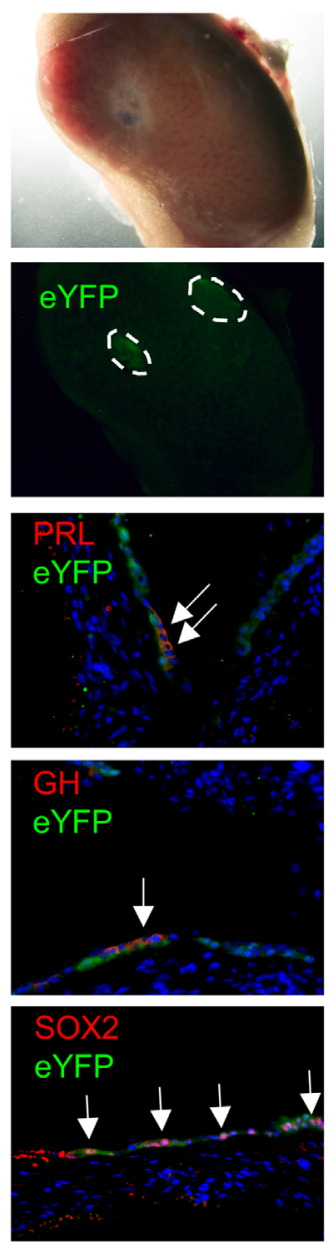

6 weeks
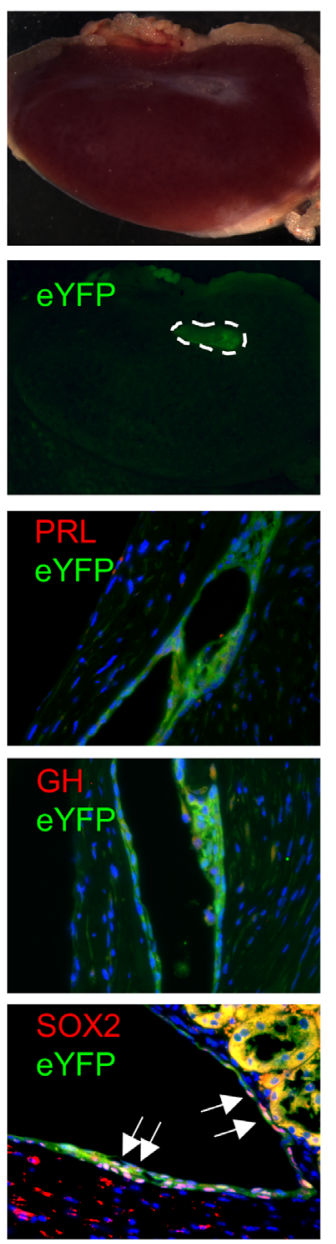

Figure 2

Differentiation capacity of pituitary organoids after in vivo transplantation. (A) Schematic representation of in vivo transplantation analysis.

Sox2CreERT2+/-;eYFPfl/- mice were injected with tamoxifen (TAM) as indicated, and eYFP+ (dense) organoids were formed from the dispersed AP cells and expanded. Organoids were grafted under the kidney capsule and analyzed 2, 4 and 6 weeks later. (B) Grafted kidneys show a fluorescent eYFP+ streak of cells (dotted line), while in the control kidney (sham) background signal was only present in the surrounding adipose tissue (arrowhead). Scale bar: $1 \mathrm{~mm}$. (C) Immunofluorescence staining of graft paraffin sections reveals some PRL $\mathrm{P}^{+}$(red)/eYFP+ (green) cells 2 weeks after grafting, as well as many SOX2+(red)/ $\mathrm{eYFP}^{+}$(green) cells (arrows). After 4 weeks, several eYFP+ (green) cells stain for GH or PRL (red) while multiple eYFP+ (green) cells still express SOX2 (red) (arrows). After 6 weeks, no hormone+ cells were observed (anymore), while SOX2+(red)/eYFP+ (green) cells remain present (arrows). Sham-grafted kidneys show no hormone- or SOX2-immunofluorescent signals. Nuclei are stained with DAPI (blue). Scale bar: 50 m. eYFP, enhanced yellow fluorescent protein; $\mathrm{GH}$, growth hormone; PRL, prolactin. 
(-/iDTR) pituitary (Fig. 3C), the latter in agreement with the findings above (Fig. 1B and Supplementary Fig. 1B). Whereas the -/iDTR-derived cultures eventually turned into complete dense organoid cultures (Fig. 3C and Supplementary Movie 1) as also found above, the GHCre/iDTR cystic organoid cultures remained cystic (Fig. 3C and Supplementary Movie 2), while a part of the mixed GHCre/iDTR cultures finally turned into dense organoids (Supplementary Fig. 3A). Whereas the dense organoids from the control pituitary were expandable for many months (Fig. 3C and above), cystic organoids stopped growing and were eventually lost after 4-8 passages (Fig. 3C and data not shown). Along the same line, dense organoids retained higher proliferative activity during passaging (as analyzed by Ki67 immunostaining) than cystic organoids (Fig. 3D), while the cell cycle inhibitors $C d k n 1 b / p 27$ and $C d k n 1 c / p 57$ were higher expressed in the cystic organoids (Supplementary Fig. 3F).
We next compared the molecular phenotype of the two organoid forms. Most cells of both dense and cystic organoids were immunopositive for the pituitary stem cell markers SOX2 and E-cadherin (Fig. 4A and above). Given the emergence of $\mathrm{SOX}_{2}+/ \mathrm{GH}^{+}$cells in the pituitary after damage (Fu et al. 2012, Willems et al. 2016), we wondered whether the cystic organoids from the DT-treated GHCre/iDTR mice were derived from these putative, activated progenitor cells. However, $\mathrm{GH}$ was not found in the cystic (neither the dense) organoids (Supplementary Fig. 3G). Moreover, the cystic organoids were not abolished by in vitro treatment with DT (Supplementary Fig. 3H), indicating that the cystic organoids did not express the $\mathrm{GH}$ promoter-induced DTR which would be expected if the originating SOX2+ cells expressed GH. The absence of DTR in the cystic organoids was further confirmed by RT-qPCR analysis (Supplementary Fig. 3I).
A
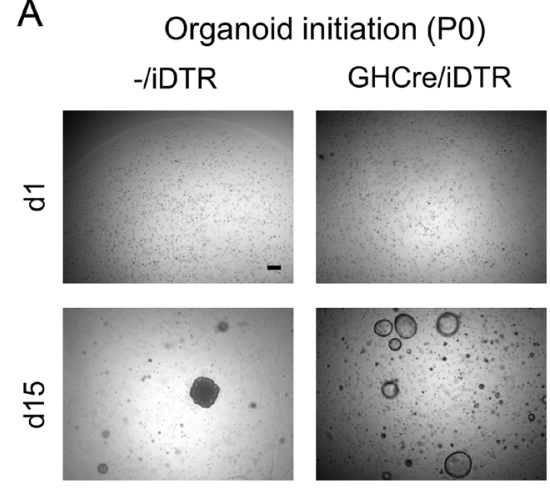

B Organoid initiation (P0)

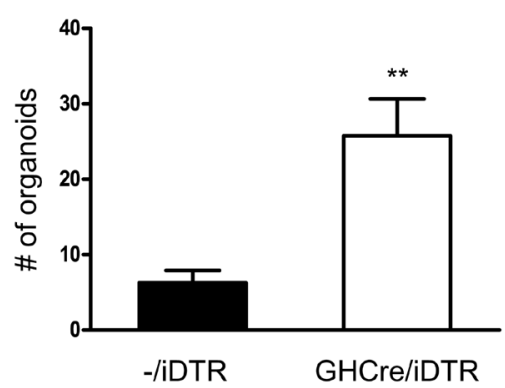
GHCre/iDTR

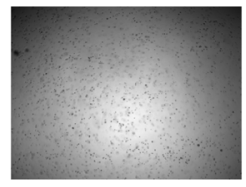

C
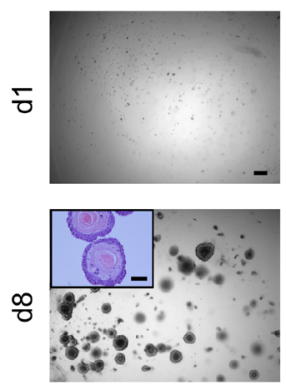

$\mathrm{P} 1$ GHCre/iDTR
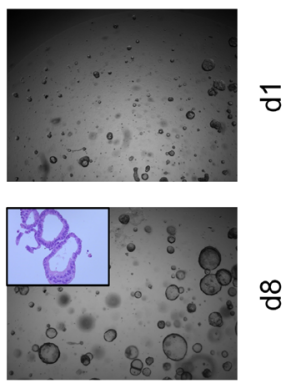

D

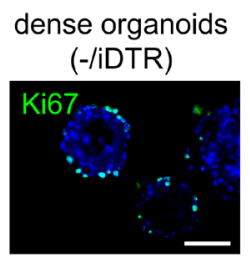

cystic organoids
P5 GHCre/iDTR
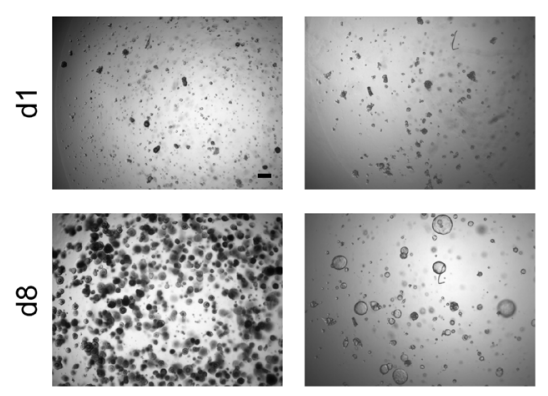

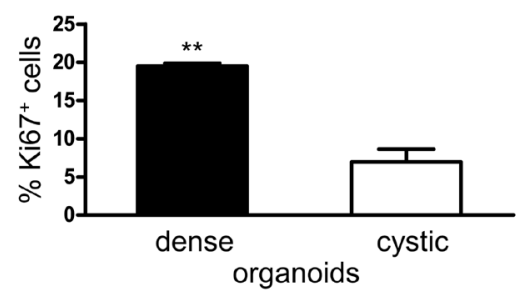

\section{Figure 3}

Organoid formation and expansion from GHCre/iDTR and -/iDTR pituitary. (A) Formation of organoids starting from dissociated AP cells of DT-injected -/iDTR (control) and GHCre/iDTR mice (P0). Representative light-microscopic pictures at seeding (d1) and at d15 are shown. Whereas predominantly dense organoids are formed in the -/iDTR AP cell cultures, GHCre/iDTR AP cell cultures give primarily rise to cystic organoids. Scale bar: $200 \mu$ m. (B) Total number of organoids (i.e. dense + cystic) formed in -/iDTR and GHCre/iDTR AP cell cultures. Bars represent mean \pm S.E.M. $(n=3)$. ** $P<0.01$. (C) Passaging and expansion of organoids from -/iDTR (control) and GHCre/iDTR pituitary, shown at P1 (d1 and d8) and P5 (d1 and d8). Organoids regrow to their original type, being either dense or cystic. After a number of passages, cystic organoids start to expand less (as shown for P5), while dense organoids keep expanding efficiently. Representative light-microscopic pictures are shown. Scale bar: $200 \mu \mathrm{m}$. Inset: H\&E staining of dense (-/iDTR) and cystic (GHCre/iDTR) organoids. Scale bar: $50 \mu \mathrm{m}$. (D) Ki67 immunofluorescence staining (green) of paraffin sections from -/iDTR dense organoids and GHCre/iDTR cystic organoids, showing higher proliferative activity in dense organoids as quantified (right). Bars represent mean \pm S.E.M. $(n=3)$. $* *<0.01$. Scale bar: $50 \mu \mathrm{m}$. 
A dense organoids cystic organoids (-liDTR)
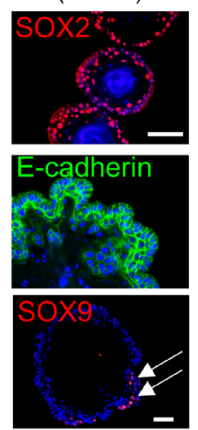

D
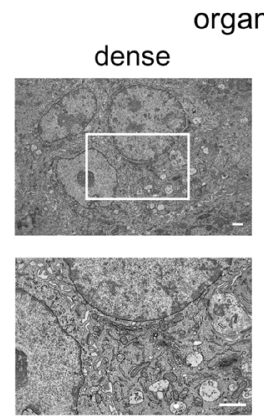

B

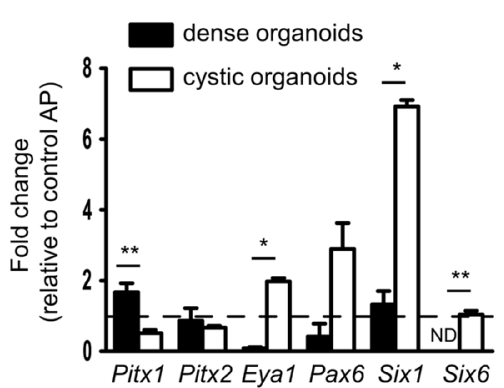

E

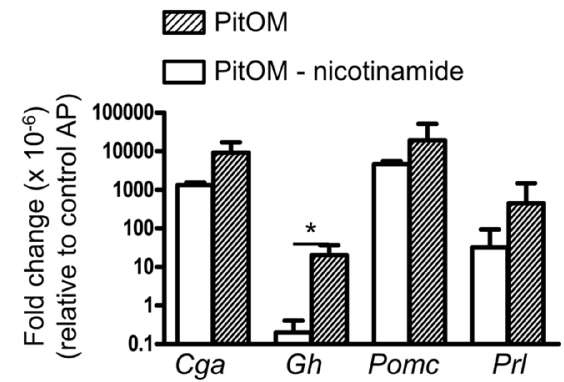

C

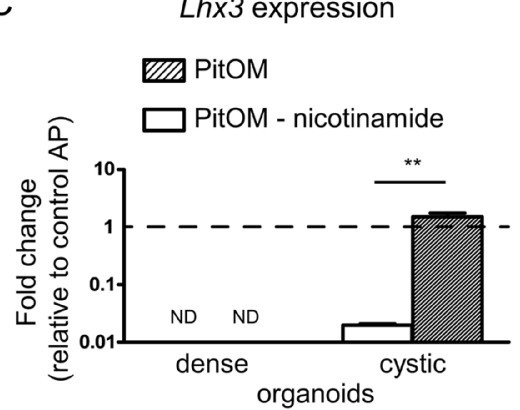

F

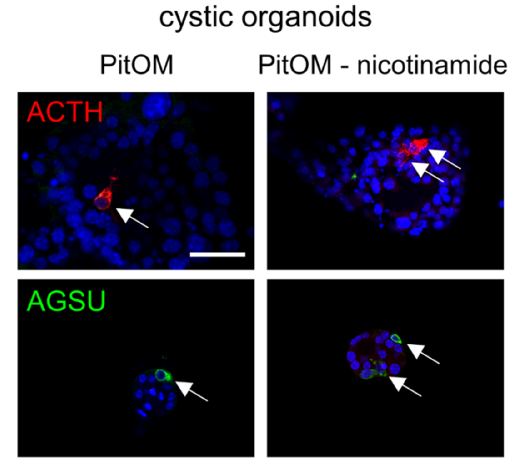

Figure 4

Cystic organoids more closely resemble the pituitary phenotype. (A) Immunofluorescence staining of SOX2 (red), E-cadherin (green) in paraffin sections and SOX9 (red) in cryosections of dense organoids as derived from undamaged -/iDTR pituitary and of cystic organoids as established from injured GHCre/iDTR pituitary. Arrows indicate SOX9+ cells. Nuclei are stained with DAPI or Hoechst (blue). Scale bar: $50 \mu$ m. (B) RT-qPCR expression analysis of early pituitary markers in dense (-/iDTR) and cystic (GHCre/iDTR) pituitary organoids (relative to expression in control AP set as 1 and indicated by dotted line). Bars represent mean \pm s.E.M. $(n=3)$. $* P<0.05 ; * * P<0.01$. (C) RT-qPCR expression analysis of $L h x 3$ in dense (-/iDTR) and cystic (GHCre/iDTR) pituitary organoids, cultured in PitOM (including nicotinamide) or PitOM without nicotinamide (relative to expression in control AP set as 1 and indicated by dotted line). Bars represent mean \pm s.E.M. $(n=3)$. ${ }^{*} P<0.01$. ND, not detectable. (D) Ultrastructural (TEM) images of dense (-/iDTR) and cystic (GHCre/iDTR) pituitary organoids showing the absence of secretory granules. Boxed areas are magnified in the lower panels. Scale bar: $2 \mu \mathrm{m}$. (E) RT-qPCR expression analysis of pituitary hormones in cystic (GHCre/iDTR) organoids, cultured in PitOM (including nicotinamide) or PitOM without nicotinamide (relative to expression in control AP). Bars represent mean \pm S.E.M. $(n=3)$. * $P<0.05$. Cga, glycoprotein hormone $\alpha$-subunit; Pomc, proopiomelanocortin (precursor of ACTH). (F) Immunofluorescent staining of ACTH (red) and AGSU (green) in cystic (GHCre/iDTR) organoids, first grown in PitOM (including nicotinamide) or PitOM without nicotinamide, and then cultured on Matrigel-coated coverslips. Cells immunopositive for ACTH or AGSU are indicated with arrows. Scale bar: $50 \mu \mathrm{m}$.

Gene expression of additional proposed pituitary stem cell markers (Sox9, Cxadr, Efnb2; Fauquier et al. 2008, Chen et al. 2009, Chen et al. 2013, Andoniadou et al. 2013, Rizzoti et al. 2013, Cox et al. 2017) was also detected in both organoid types (Supplementary Fig. 4A). Cxadr showed the most pronounced enrichment vs AP tissue, whereas Sox9 was not enriched. In line with the latter observation, only a limited number of cells in the organoids (of both types) were found to express SOX9 at the protein level, and SOX9+ cells were not detected in every organoid or organoid section (Fig. 4A and Supplementary Fig. 4B). Expression of transcription factors involved in the earliest phases of pituitary embryogenesis (i.e. Pitx1, Pitx2, Eya1, Pax6, Six1, Six6; Vankelecom 2010, 2012) was found in both dense and cystic organoids, however showing different profiles (Fig. 4B). Whereas the dense organoids (from -/iDTR pituitary) exhibited significantly higher expression of Pitx1 (also enriched as compared to the whole AP, Fig. 4B), cystic organoids (from GHCre/iDTR pituitary) showed significantly upregulated expression of Eya1, Six1 and Six6 (Fig. 4B), the latter representing a direct downstream target of SOX2 in the developing pituitary and required for proper proliferation and differentiation of embryonic pituitary progenitor cells (Goldsmith et al. 2016). Expression of $L h x 3$ and Prop 1 , transcription factors active in embryonic (but also adult) pituitary cells, was only detectable in cystic organoids although at low level when compared to adult AP (Fig. 4C and Supplementary Fig. 4C). Gene expression of pituitary hormones was detected in both organoid types, but again at very low levels as compared to AP tissue (Supplementary Fig. 4D). In line with the apparent more pronounced pituitary 
phenotype of cystic organoids (i.e. higher expression of most embryonic transcription factors), expression of hormone genes, in particular Cga and Pomc (encoding aGSU and ACTH, respectively), was found higher in cystic than dense organoids (Supplementary Fig. 4D). However, the very low mRNA levels are not translated into detectable protein given the absence of hormone immunoreactivity (in both organoid types) as reported above (Fig. 2 and Supplementary Figs 2, 3G, and data not shown). In accordance, transmission electron microscopy (TEM) did not find secretory granules in the cells of both organoid types, thereby further documenting their immature phenotype (Fig. 4D). Secretory granules are typically observed in the hormone-producing cells of the AP but are not present in the putative stem cells of the gland (Supplementary Fig. 4E). Intriguingly, removing nicotinamide from the organoid culture medium PitOM resulted in a significant increase in $L h x 3$ expression in the cystic organoids, even to levels comparable to the adult AP, while Prop1 levels were not significantly upregulated (Supplementary Fig. 4C). Their expression remained absent in the dense organoids after nicotinamide withdrawal (Fig. 4C and Supplementary Fig. 4C). Removal of nicotinamide from PitOM also induced higher expression (significantly or trend) of hormone genes in the cystic organoids, but not in the dense organoids (Fig. 4E and data not shown). To assess hormonal differentiation capacity of the cystic organoids, we cultured the organoids on Matrigel-coated coverslips (Fauquier et al. 2008, Chen et al. 2009), which failed to induce in vitro differentiation of dense organoids (see above). Since mRNA levels of Pomc and $C g a$ were highest in cystic organoids (Fig. 4E and Supplementary Fig. 4D), we analyzed specifically these hormones at the protein level. $\mathrm{ACTH}^{+}$cells were detected in the cystic organoids $(0.1 \pm 0.07 \%$ and $1.3 \pm 0.4 \%$ of the cells after pre-growth in PitOM with nicotinamide and without nicotinamide, respectively; $n=3, P=0.10$ ) (Fig. 4F). AGSU+ cells were also observed in both conditions $(0.5 \pm 0.3 \%$ and $0.6 \pm 0.4 \%$, respectively; $n=3, P=0.86)$. In agreement with the presence of $\mathrm{ACTH}^{+}$and AGSU+ cells in the cystic organoids after applying the differentiation protocol, gene expression of the receptors for the cognate hypothalamic regulatory hormones (i.e. Crhr1 and Gnrhr) was also detected (Supplementary Fig. 4F). Finally, we transplanted cystic organoids under the kidney capsule of NOD/SCID mice. However, we could not detect a graft as analyzed 4 weeks later (data not shown), likely due to the insufficient number of cystic organoids transplanted given their more restricted expandability.
Taken together, the organoids as developed from damaged pituitary (which harbors activated stem cells) differ from the organoids established from basal, undamaged pituitary and more closely resemble the (immature) pituitary phenotype.

\section{Dense and cystic organoids exhibit a distinct epithelial phenotype}

To further decipher the molecular differences between the dense and cystic pituitary organoid types, RNA-sequencing (RNA-seq) analysis was performed. GO examination revealed keratinization as one of the top enriched biological processes in the dense vs cystic organoids (Fig. 5A). Keratin (Krt) gene expression phenotype clearly differed between the cystic and dense organoids (Fig. 5B). Interestingly, the $K r t$ expression profile in the cystic organoids clustered with the expression in the SOX2+ stem cells (as isolated from both injured and non-injured Sox2eGFP pituitary), whereas the expression pattern in the dense organoids was clearly different (Fig. 5B). In accordance, also the expression of the embryogenesisrelated factors in the cystic organoids correlated with the expression in the SOX2+ cells (Supplementary Fig. 4G). The latter RNA-seq data further validated the differential expression between dense and cystic organoids as found above by RT-qPCR (Fig. 4B). Regarding the $K r t$ expression profile, a clear dichotomy between cystic and dense organoids was observed for Krt8/Krt18 and Krt5/Krt14 (Fig. 5B, boxed) as confirmed by RT-qPCR (Supplementary Fig. 5A). Krt8 (encoding for cytokeratin 8, CK8) and Krt18 (CK18), typical markers of simple non-squamous (columnar or cuboidal) epithelium, were higher expressed in the cystic vs dense organoids (Fig. 5B and Supplementary Fig. 5A). Krt5 (CK5) and Krt14 (CK14), typical markers of stratified squamous epithelium, were strongly upregulated in the dense vs cystic organoids. Also other markers of squamous epithelium (Trp63, Lor, Tgm1; Candi et al. 2006) were found upregulated in the dense vs cystic organoids (Supplementary Fig. 5B). We further analyzed the dichotomic keratins at the protein level and found in the cystic organoids intense immunopositivity for CK8/CK18 but not for CK5/CK14, whereas the dense organoids strongly expressed CK5 and CK14 and showed only low immunoreactivity for CK8/CK18 (Fig. 5C).

These different epithelial phenotypes were further supported by whole-organoid two-photon microscopy scanning (Supplementary Movies 1 and 2) and by TEM (Fig. 5D). Cystic organoids were composed of tightly 
A

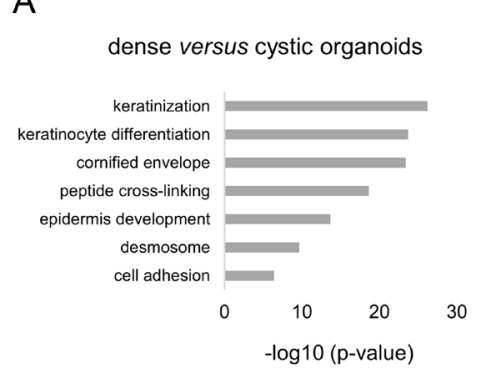

B

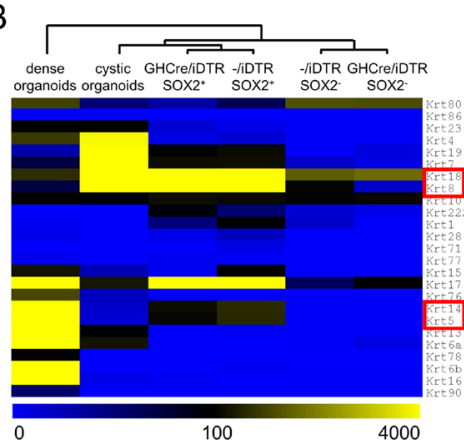

C

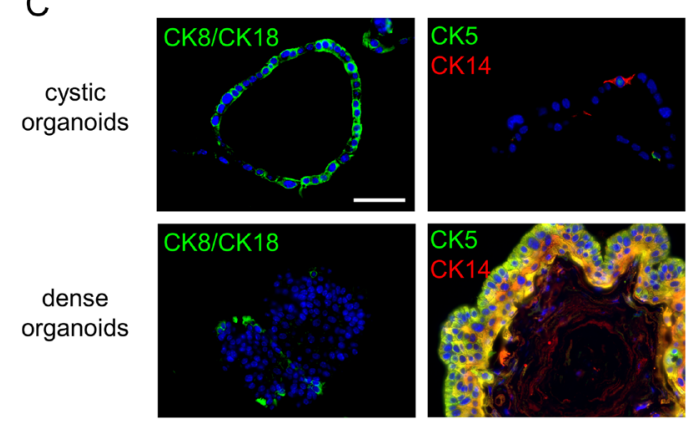

D

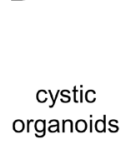

overview

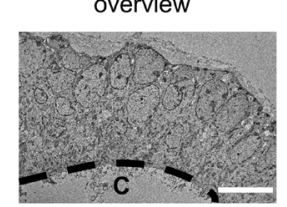

cell junctions
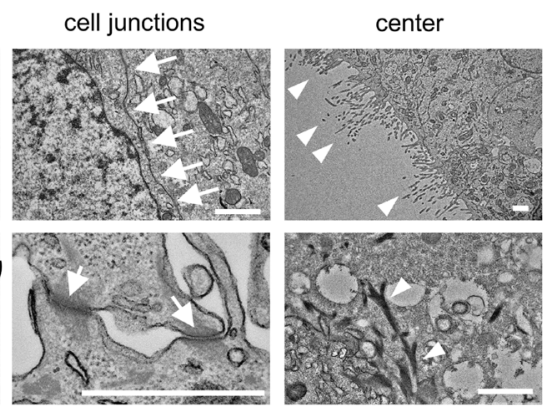

Figure $\mathbf{5}$

Dense and cystic pituitary organoids show a different epithelium identity. (A) Overview of a number of enriched biological processes (and their significance level, denoted as the -log10 of the corresponding $P$-value) in dense organoids versus cystic organoids, as identified by GO analysis. (B) Heatmap from RNA-seq data showing the differential expression of keratin ( $K r t$ ) genes (from high expression in yellow to low expression in blue) in dense (-/iDTR) and cystic (GHCre/iDTR) organoids as compared to pituitary SOX2+ and SOX2- cells (isolated as SOX2eGFPpos and SOX2eGFPneg cells by FACS from -/iDTR undamaged or GHCre/iDTR damaged pituitary). (C) Immunofluorescence staining of CK8/CK18 (green), CK5 (green) and CK14 (red) in paraffin sections of cystic and dense pituitary organoids. Nuclei are stained with DAPI (blue). Scale bar: $50 \mu \mathrm{m}$. (D) Ultrastructural (TEM) images of cystic and dense pituitary organoids. Upper panels: TEM analysis of cystic organoids showing tight cell junctions (arrows) and microvilli facing the lumen (arrowheads). Lower panels: TEM analysis of dense organoids showing desmosomal cell junctions (arrows) and signs of keratinization in the center (arrowheads). Scale bar: $10 \mu \mathrm{m}$ in overview picture, $1 \mu \mathrm{m}$ in other pictures. C, center (lumen in cystic organoids).

connected cells with polarized character and presence of microvilli selectively at the luminal side of the organoids, all typical of simple columnar/cuboidal epithelium (Fig. 5D). In contrast, in the dense organoids, cell membranes were not tightly connected but contained many desmosomes, commonly found in squamous epithelium (Fig. 5D). Moreover, TEM revealed keratin depositions in the center of the dense organoids, in line with the upregulated keratinization process as found by GO analysis, and as further confirmed by pancytokeratin immunostaining (Supplementary Fig. 5C).

\section{Application of the organoid model as a new tool toward pituitary stem cell exploration}

Organoids are valuable models to study tissue stem cell biology (Clevers 2016). Therefore, we addressed the question whether pituitary organoids could provide a model to explore and reveal new aspects of the putative pituitary stem cells, in particular regarding identity, behavior and regulation.

\section{Pituitary organoids reveal keratin and Prrx stem cell marker expression and behavior}

Obtaining strikingly divergent cystic and dense organoids from pituitary raised the question whether different stem cell (sub-)populations or phenotypes/states exist in the pituitary gland. We used the prominent dichotomy of keratin expression in the two organoid types to approach this question by analyzing the expression of CK8/CK18 and CK5/CK14 in the pituitary in situ. CK8/CK18 immunoreactivity highly colocalized with $\mathrm{SOX}^{+}$cells in the proposed stem cell niches (marginal zone and parenchymal clusters) of normal and damaged pituitary (Fig. 6A). In contrast, CK5 and CK14 immunoreactivity was not or only scarcely detected in both pituitary conditions (Fig. 6B).

We hypothesize that the CK8/CK18/SOX2+ cells, once activated as occurring after the $\mathrm{GH}^{+}$cell ablation damage (Fu et al. 2012, Willems et al. 2016), readily form the large cystic organoids as observed, while in the undamaged resting pituitary, the majority of these CK8/CK18/SOX2+ cells are quiescent with only some 
A
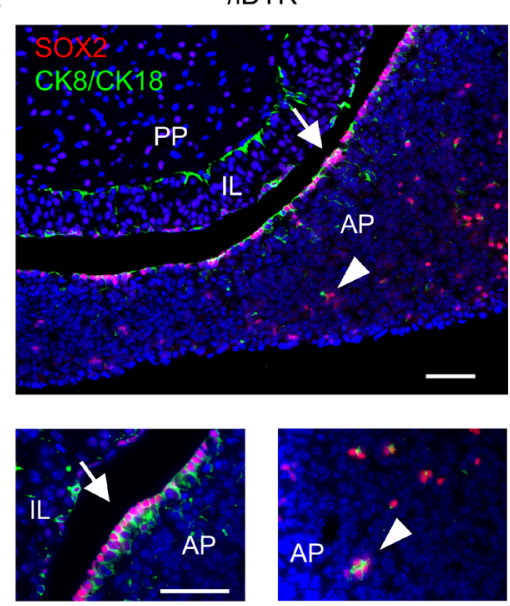

B

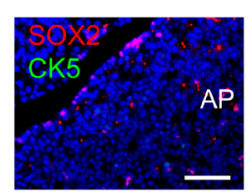

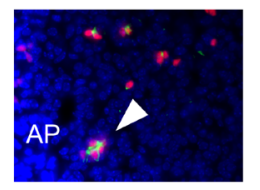

-/iDTR

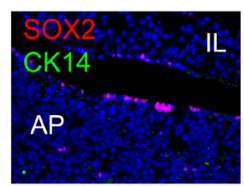

GHCre/iDTR
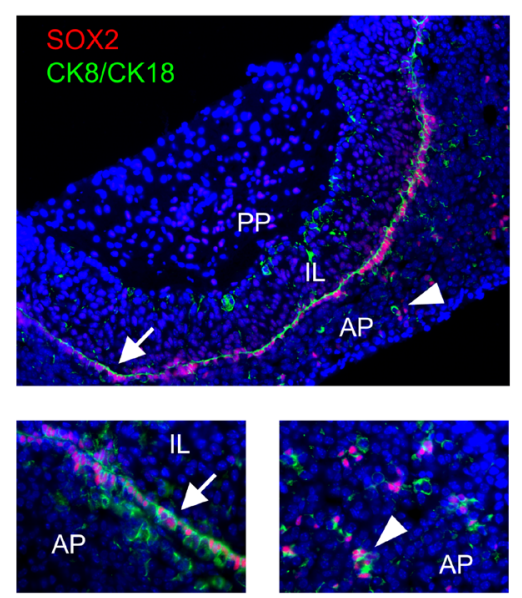

GHCre/iDTR

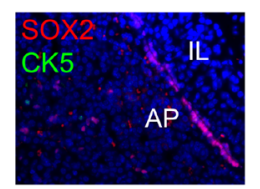

C
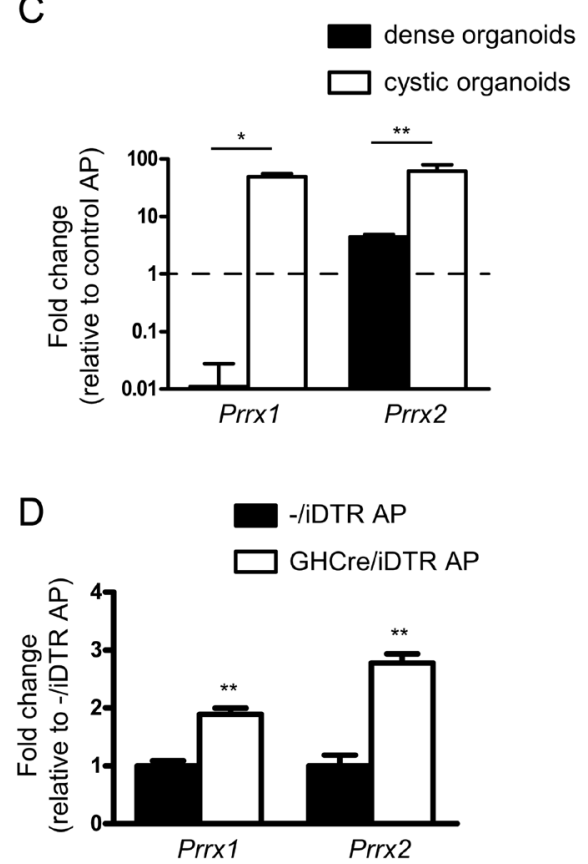

\section{Figure 6}

Expression of distinct keratins in the pituitary SOX2+ cell compartment and of Prrx1/2 in organoids and pituitary after damage. (A) Immunofluorescent staining of SOX2 (red) and CK8/CK18 (green) in -/iDTR and GHCre/iDTR pituitary after DT injection (d11). CK8/CK18 signal is found in SOX2+ cells in both the marginal zone of the AP (arrows) and in parenchymal clusters (arrowheads), as shown in overview and magnified pictures (lower panel). AP, anterior pituitary; CK, cytokeratin; IL, intermediate lobe; PP, posterior pituitary. Scale bar: $50 \mu \mathrm{m}$. (B) Immunofluorescent staining of SOX2 (red) and CK5 or CK14 (green) in -/iDTR and GHCre/iDTR pituitary after DT injection (d11). CK5+ and CK14+ signals were not, or only scarcely detected. (C) RT-qPCR expression analysis of Prrx 1 and Prrx2 in dense (-/iDTR) and cystic (GHCre/iDTR) pituitary organoids (relative to expression in control AP set as 1 and indicated by dotted line). ${ }^{*}<<0.05 ; * * P<0.01$. (D) RT-qPCR expression analysis of Prrx 1 and Prrx2 in the AP from GHCre/iDTR and control -/iDTR mice (relative to expression in -/iDTR AP set as 1). Bars represent mean \pm s.E.M. $(n=3)$. $* * P<0.01$.

sporadic cells (stochastically) activated in a sufficient way to form (small) cystic organoids (Fig. 1A). That activation of the pituitary stem cells may play a role in the cystic organoid formation, is further supported by the following findings. First, abundant organoids, all displaying a cystic phenotype, were obtained from neonatal pituitary (Supplementary Fig. 6A) whose stem cell compartment is more activated (Gremeaux et al. 2012). Second, organoids that were generated from pituispheres established from undamaged adult AP were of cystic phenotype (Supplementary Fig. 6B). Pituisphere formation involves (proliferative) activation of the stem cells by the specific culture conditions used (Chen et al. 2005, 2009, Fauquier et al. 2008, Garcia-Lavandeira et al. 2009, Fu et al. 2012). How the CK5/CK14/SOX2+ dense organoids originate is at present not clear given the high scarcity of CK5 and CK14 immunoreactivity in the adult AP, although it should be noted that Krt5 and Krt14 mRNA was clearly detectable in the SOX2+ cell population and found enriched when compared to the SOX2- cells (Fig. 5B). The Krt5/Krt14expressing $\mathrm{SOX}^{+}{ }^{+}$cells may start to translate the mRNA at organoid formation. Another possibility is that
CK8/CK18/SOX2+ cells switch epithelial phenotype (to $\mathrm{CK} 5 / \mathrm{CK} 14 / \mathrm{SOX}^{+}{ }^{+}$) in vitro in the context of the organoidforming conditions. We occasionally observed cystic organoids that transformed into dense organoids when other dense organoids were present in the vicinity, thereby suggesting an inductive (paracrine) effect of the dense organoid to cause the cystic forms to switch epithelial phenotype (Supplementary Fig. 6C). Accordingly, mixed CK8/CK18 ${ }^{+} \mathrm{CK}^{+}$organoids were sporadically seen (Supplementary Fig. 6D).

PRRX1 and PRRX2 have recently been advanced as plausible stem cell markers in the pituitary (Vankelecom 2010, Higuchi et al. 2014, Cox et al. 2017). We detected strong upregulation of Prrx 1 and Prrx 2 in the damaged pituitary-derived cystic organoids as compared to the dense organoids (Fig. 6C; also observed in the RNA-seq analysis, data not shown). Taking this finding back to the pituitary in vivo, we found that Prrx1 and Prxx2 gene expression levels were upregulated in the AP after damage (Fig. 6D), thus suggesting a role in stem cell activation upon injury. This finding highlights the power of the pituitary organoid models, and in particular their comparison, to unravel the 
behavior of stem cell-associated markers in the pituitary as occurring during damage and regeneration.

\section{Pituitary organoids reveal peculiarities of the stem cell-regulatory WNT/LGR pathway}

The WNT/LGR pathway is known to play a key role in stem cell regulation in many tissues, including their selfrenewal/maintenance and activation upon injury (Barker et al. 2007, Huch et al. 2013b). To start investigating the role of WNT/LGR in pituitary stem cell regulation, we used our organoid system and tested the influence of this pathway in pituitary organoid initiation (P0) and expansion from both normal and damaged gland. Omission of WNT3A did not affect organoid development (P0), neither in the control (-/iDTR) nor in the GHCre/iDTR conditions (Fig. 7A and Supplementary Fig. 7A). In contrast, removal of RSPO1 reduced organoid development in the GHCre/iDTR culture, while not overtly changing the organoid formation efficiency in the -/iDTR condition (Fig. 7A and Supplementary Fig. 7A). Addition of the WNT pathway inhibitor IWP2 (which blocks endogenous WNT ligand palmitoylation as needed for secretion) significantly reduced organoid formation efficiency in the GHCre/iDTR condition (Fig. 7A and Supplementary Fig. 7A). Also, addition of another WNT inhibitor, XAV939 (which stabilizes the canonical WNT-inhibitory protein AXIN2), efficiently blocked the formation of cystic (GHCre/iDTR) organoids, while dense (-/iDTR) organoids still formed (Supplementary Fig. 7B). WNT3A could not rescue the formation of organoids in the IWP2-treated cultures (Fig. 7A and Supplementary Fig. 7A).

Also for further expansion and passaging of the organoids, WNT3A was found not essential (in both dense and cystic organoid cultures), whereas RSPO1 was needed to maintain the growth of the cystic organoids over different passages (Fig. 7B). IWP2 impaired cystic organoid expansion, which is only slightly rescued by WNT3A (Fig. 7B). XAV939 also inhibited the expansion of cystic organoids (Supplementary Fig. 7C). Intriguingly, the dense organoids kept expanding regardless of the presence of RSPO1 (and WNT3A), and expansion was only minimally affected by IWP2 or XAV939 (Fig. 7B and Supplementary Fig. 7C).

The dense and cystic organoids thus showed distinct WNT/RSPO growth responsiveness. To investigate whether these functional characteristics correlated with molecular features, we analyzed the endogenous expression of WNT ligands in both organoid types. RNA-seq analysis pointed to an overall higher expression of WNT ligands in the dense organoids (Fig. 7C; validated for a selection by
RT-qPCR in Supplementary Fig. 7D) which may be in line with their independency of exogenous WNT3A.

The WNT-amplifier RSPO1 is known as the typical ligand for LGR5. We found high expression of Lgr5 in the cystic organoids (i.e. obtained from the damaged GHCre/iDTR pituitary) while expression is virtually absent in the dense organoids (from control pituitary) (Fig. 7D), in line with the need for RSPO1 in cystic organoid culturing and its redundancy in dense organoid growth. In analogy with Lgr5 gene expression in the cystic organoids, expression was also found upregulated in the GHCre/iDTR pituitary in situ after DT-induced damage (as analyzed by RT-qPCR and RNAscope in situ hybridization) (Fig. 7E and Supplementary Fig. 7E). Emergence of LGR5 expression after damage has also been reported in other tissues with low turnover rate (such as the liver and pancreas; Huch et al. 2013a,b). Using the triple transgenic GHCre/iDTR/Lgr5lacZ mouse model, we observed some lacZ ${ }^{+}$cells (weak signal) arising in the pituitary after damage, some of them in the proposed marginal zone stem cell niche (Supplementary Fig. 7F). Moreover, when AP cells from this mouse model were cultured in organoid-forming culture conditions, a number of lacZ $\mathrm{Z}^{+}$organoids were detected (as analyzed at P2; Supplementary Fig. 7G). Not all organoids were lacZ ${ }^{+}$suggesting that (i) not all organoids are derived from the Lgr $5^{\text {lacZ+ }}$ cells emerging after damage; (ii) that the Lgr5lacZ+ signal is lost upon organoid culture and expansion or (iii) the efficiency of the Lgr5lacZ reporter system is low in the pituitary and the organoids (as supported by the only weak signals appearing in the pituitary in situ). Expression of the LGR5 ligand Rspo1 was also found upregulated in the injured pituitary (Fig. 7E) but was not detected in the organoid cultures (data not shown), explaining why addition of exogenous RSPO1 is needed for the (cystic) organoids to develop and grow. Taken together, our new pituitary organoid models may lead to a valuable tool to study the regulation of putative pituitary stem cells in both normal and activated condition, as exemplified here by revealing particular WNT/LGR pathway aspects.

Finally, since the dense organoid cultures were found to thrive without addition of WNT3A and RSPO1, we also tested whether other (growth) factors were indeed needed for the long-term expansion, and removed FGF2/8/10, $\mathrm{SHH}$, Noggin and cholera toxin (Table 1; the depleted medium further termed 'EASI' medium referring to the remaining core factors, viz. EGF, A83-01, SB202190 and IGF1). Surprisingly, the dense organoids remained as expandable in EASI medium as in the original PitOM 
A

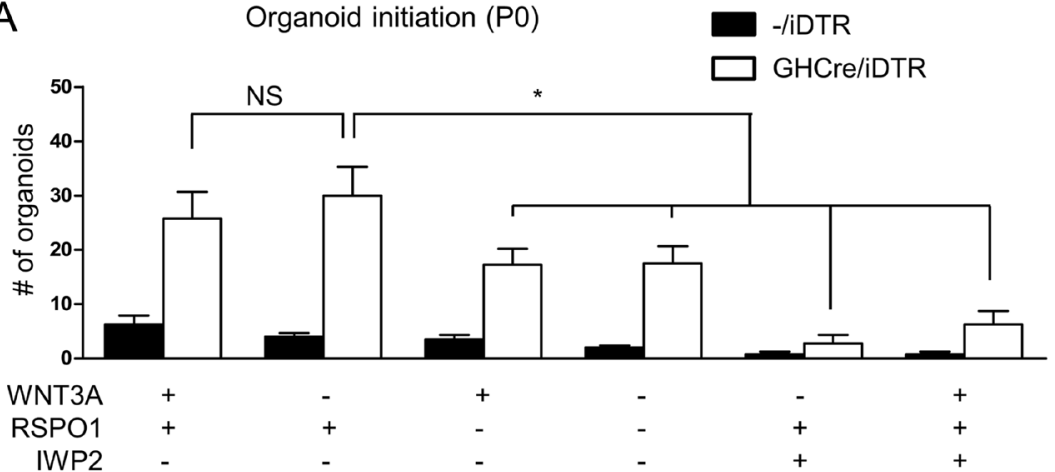

B

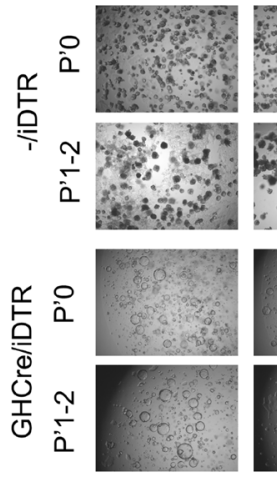

WNT3A +

RSPO1 +

IWP2

C

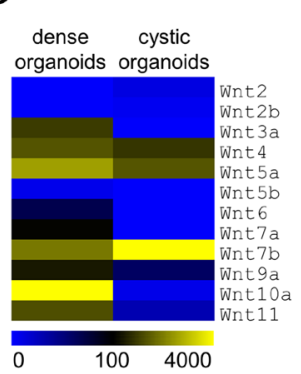

D

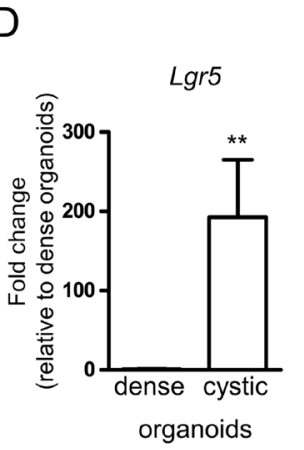

E
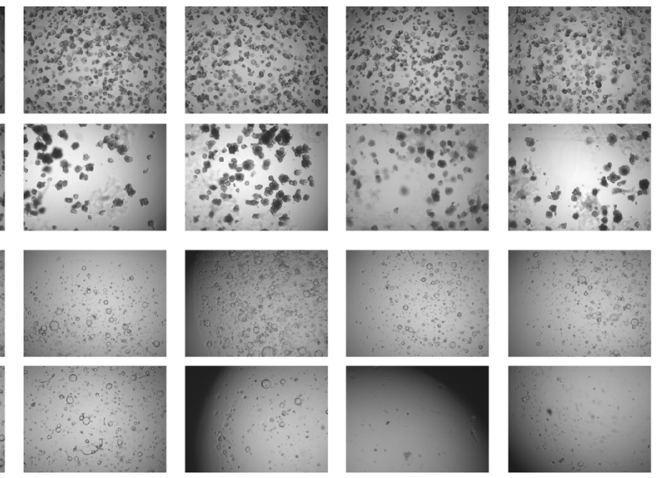

$+$
E $\square$ GHCre/iDTR AP

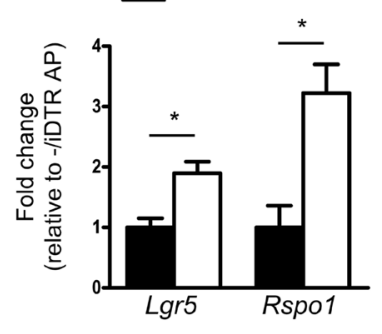

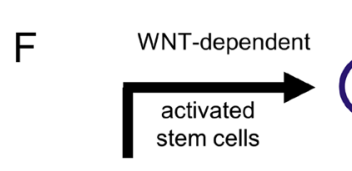

pituitary stem cells

$\because \because \because$

?

I

WNT-independent

rare squamous cells/ epithelial transition/

back in development

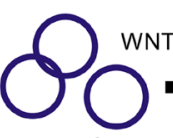

cystic

organoids
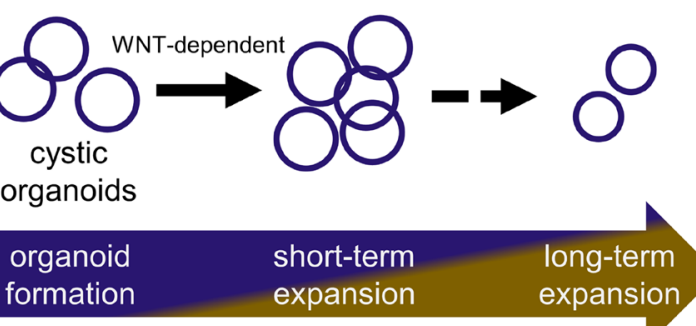

CK8/CK18/SOX2+

CK5/CK14/SOX2+ 
(Supplementary Fig. 8A). EGF proved to be an essential, but not sufficient growth factor for this dense organoid expansion (Supplementary Fig. 8A), indicating that one or more of the other factors still present in the EASI medium are necessary for optimal organoid growth and expansion. A83-01, the small-molecule inhibitor of TGFB signaling, was found to be required for optimal expansion, while adding TGFB1, in the absence of its inhibitor, further compromised (i.e. arrested) organoid growth (Supplementary Fig. 8B). Finally, we found that EASI medium was even sufficient for the initial formation (P0) of dense (but not cystic) organoids (Supplementary Fig. 8C and data not shown). For the formation of cystic organoids, several PitOM components were found to be crucial, including EGF (Supplementary Fig. 3D), the p38 inhibitor SB202190 and cholera toxin (Supplementary Fig. 8D). Together, these findings may provide hints toward regulatory pathways of the putative pituitary stem cells.

In conclusion, our new pituitary organoid system presents itself as a promising tool toward studying and deciphering pituitary stem cell biology, including identity, behavior and regulation.

\section{Discussion}

In the present study, we report the first development of organoids starting from (mouse) pituitary. Organoids typically develop from a tissue's stem cells using a cocktail of growth and regulatory factors active in the tissue stem cell niche. Since knowledge on pituitary stem cell niche factors is almost non-existing, we tested manifold stem cell regulators, together with growth and signaling factors important in pituitary embryogenesis (Vankelecom 2010, 2012, Boretto et al. 2017, Cox et al. 2017). By trial-anderror method, we eventually defined culture conditions enabling pituitary organoid formation and expansion. The organoids are derived from pituitary SOX2+ cells which have been shown to include pituitary stem cells (Fauquier et al. 2008, Chen et al. 2009, Garcia-Lavandeira et al. 2009, Fu et al. 2012, Gremeaux et al. 2012, Andoniadou et al. 2013, Chen et al. 2013, Rizzoti et al. 2013, Higuchi et al. 2014,). The low proportion of organoid-forming SOX2+ cells, as observed here, is in accord with the low proportion of colony-initiating $\mathrm{SOX}^{+}$(stem) cells as reported before (Gaston-Massuet et al. 2011, Andoniadou et al. 2013). Several possible explanations may apply for this low number, separately or in combination: (i) colony-, pituisphere- and organoid-growing stem cells (i.e. susceptible to proliferative activation) represent only a small subpopulation of the predominantly quiescent SOX2+ (stem) cells; (ii) not all pituitary SOX2+ cells are stem cells; (iii) isolation by FACS is known to cause detrimental cell shearing and friction, leading to an underestimation; (iv) the culture conditions are not ideal (yet) to instigate many stem cells to form pituispheres, colonies or organoids. The stemness phenotype of the organoid-forming cells is, in addition to expression of several pituitary stem cell markers, supported by their self-renewal capacity since they can regrow organoids in subsequent passages, and their development from pituispheres, shown by several groups to be (clonally) derived from (SOX2+) stem cells (Chen et al. 2005, 2009, Fauquier et al. 2008, GarciaLavandeira et al. 2009, Andoniadou et al. 2013, Rizzoti et al. 2013). Further circumstantial evidence is provided by the findings that organoid abundance follows the known pituitary stem cell activation status being higher in the neonatal pituitary (Gremeaux et al. 2012) and after damage (Fu \& Vankelecom 2012, Fu et al. 2012, Willems et al. 2016). Applying the developed organoid protocol to our previously established pituitary damage-andregeneration model yielded additional interesting findings. Organoid morphology clearly differed, being cystic from the damaged gland and dense from the undamaged gland. Further remarkably, the dense organoids, once established, could be long-term propagated, whereas the cystic organoids were more limited in expandability. Several possible reasons may account for the latter observation. Pituitary stem cells may, once activated, have restricted self-renewal capacity and get exhausted after a couple of division rounds (as also observed for hair follicle and neural stem cells; Sierra et al. 2015, Lay et al. 2016). Of note, we recently found that prolonging the period of pituitary damage infliction (10 consecutive days of DT treatment instead of 3 days; Willems et al. 2016) annihilated the regeneration, which may also be due to exhaustion of the pituitary stem cells being continuously triggered to self-renew because of the unceasing and longer damage. Alternatively, efficient long-term expansion of cystic organoids may need additional still unidentified growth or regulatory factors. For example, some of the pituitary stem cell-activating factor(s) emerging upon damage in vivo may not be present (since still unknown) in the organoid medium, and the stem cells may thus lose their activated status during culture. Another culprit may be the WNT pathway: cystic organoids show lower endogenous expression of many WNT ligands as compared to dense organoids which may be needed for efficient expandability. However, although high 
WNT ligand expression in the dense organoids may explain their independency of exogenous WNT/RSPO factors, dense organoids can expand even in the presence of the inhibitor (IWP2) of endogenous WNT ligand production. On the other hand, the exogenous WNT activation as applied may lead to premature exhaustion of expansion capacity, decrease in stemness or induction of stem cell quiescence (Gregorieff et al. 2015, De JaimeSoguero et al. 2017) in the cystic organoid cultures, the latter in line with the strong expression of the cell cycle inhibitors $p 27$ and p57. Interestingly, p57 expression during pituitary embryogenesis indicates growth arrest of the embryonic progenitor cells and start of their differentiation (Bilodeau et al. 2009). In organoid cultures from undamaged pituitary, some cystic organoids were also formed, but structures remained generally small (in contrast to the large cystic organoids from the damaged gland) and did not, or only minimally, propagate in culture. These (small) cystic organoids may originate from some sporadic, stochastically activated stem cells (e.g. primed for self-renewal) but only activated to a low degree, explaining the small size and non-expandability of these organoids. When starting from neonatal pituitary, known to house a more activated and abundant stem cell population as compared to adult pituitary (Gremeaux et al. 2012), an increased number of (cystic) organoids form, thereby supporting the hypothesis that cystic organoid formation relies on activation of the stem cell compartment. In addition, when starting from pituispheres established from adult pituitary (which are composed of activated stem cells; Chen et al. 2005, 2009, Fauquier et al. 2008, Garcia-Lavandeira et al. 2009, Fu et al. 2012), also cystic organoids develop.

RNA-seq analysis revealed a further, unexpected difference between both organoid types. Whereas cystic organoids possess characteristics of simple columnar/ cuboidal epithelium, dense organoids display features of stratified squamous epithelium. The underlying keratin expression profile guided us to specific keratins, i.e. CK8/ CK18, that were found to mark the pituitary SOX2+ stem cells in vivo as present in the proposed marginal zone and parenchymal stem cell niches. Moreover, the profiling showed that the cystic organoid type is closer to a pituitary phenotype than the dense organoid form, further supported by the pituitary transcription factor and hormone gene expression patterns, being generally higher in the cystic than the dense organoids. Our findings altogether support the idea that cystic organoids are derived from $\mathrm{CK} 8 / \mathrm{CK} 18 / \mathrm{SOX} 2^{+}$(stem) cells of the pituitary that, once activated as occurring after $\mathrm{GH}^{+}$cell ablation damage, readily form organoids (cystic in nature) that are expandable till the stem cells get exhausted (Fig. 7F). These organoids retain a stemness or immature (embryonic) pituitary phenotype during expansive culture.

At present, the origin of the dense organoids remains more of a conundrum. Although CK5/CK14/SOX2immunoreactive cells were not observed in the pituitary in situ, Krt5 and Krt14 gene expression was detected and enriched in the $\mathrm{SOX}_{2}+$ cell population (vs the SOX2cell population). Translation to protein may be initiated in vitro in those (quiescent, squamous) $\mathrm{SOX} 2+$ cells that generate the dense organoids (Fig. 7F). Another possible explanation is that the dense organoids originate from certain (specific?) CK8/CK18/SOX2+ cells that undergo an epithelial switch ('metaplasia') in vitro in the context of the organoid-forming conditions (Fig. 7F), as suggested by our observation that cystic organoids can sometimes convert into dense organoids and that hybrid organoids are sporadically found that express both CK8/CK18 and CK5. Conversion of epithelium type is well known to occur during embryonic development of several epithelial tissues, as well as during tumorigenesis (Yu et al. 2005, Moll et al. 2008, Van Keymeulen et al. 2011). Interestingly, differential expression of cytokeratins has also been described in the human pituitary, in particular in Rathke's cleft cysts vs craniopharyngiomas. CK8 is found in Rathke's cleft cysts (but not or only diffuse in craniopharyngiomas; Tateyama et al. 2001, Xin et al. 2002) which are considered to represent remnants of Rathke's pouch, the embryonic anlage of the pituitary, highly composed of stem/ progenitor cells. That CK8 may thus also mark stem cell-linked cells in the human pituitary which further underscores the relevance of our new organoid model. On the other hand, adamantinomatous craniopharyngioma, a tumor that is most plausibly initiated by pituitary SOX2+ cells (Andoniadou et al. 2013), shows CK5/CK6/CK14 expression and a predominant squamous identity (Tateyama et al. 2001, Xin et al. 2002, Buslei et al. 2007).

Finally, we speculate that the dense organoids may represent a more primitive state of the pituitary (Fig. 7F), in which the pituitary stem cells may even revert to the phase of oral ectoderm, a stratified squamous epithelium that lines the stomodeum and gives rise to Rathke's pouch (Lanctôt et al. 1997, Hughes \& Chuong 2003). In line with this hypothesis, Pitx1 and Pitx2, transcription factors involved in early oral and pituitary development (Gage \& Suh 1999, Charles et al. 2005), are both higher expressed in the dense organoids than the cystic organoids (significantly for Pitx1), while the more 
pituitary-typical marker $\operatorname{Lh} x 3$ is not detected in the dense organoids. In further support, Krt76, expressed in the stratified squamous epithelium of the hard palate and the gingiva (Moll et al. 2008) which also develop from the stomodeum (Lanctôt et al. 1997), is exclusively detected in the dense organoids (Fig. 5B). However, SOX9, which is an established marker of pituitary stem cells (Fauquier et al. 2008, Chen et al. 2009, Garcia-Lavandeira et al. 2009, Andoniadou et al. 2013, Rizzoti et al. 2013) but not yet expressed in the early oral ectoderm, is detected in the dense organoids although only in some rare cells. SOX9 arises during pituitary embryogenesis after SOX2 (e12.5-e14.5; Fauquier et al. 2008, Rizzoti et al. 2013), thus suggesting that the dense organoids are not pure oral ectodermal structures but show already some pituitary commitment. A similar expression pattern of SOX9 in the cystic organoids (both at the protein and mRNA level) (as well as of Cxadr mRNA) supports the pituitary-kindred relationship between the cystic and dense organoids. Of note, it has been reported before that SOX9 is initially absent in stem cell-derived pituispheres (Fauquier et al. 2008, Rizzoti et al. 2013) but only emerges 6-7 days later. Thus, the organoids appear to mainly remain in the early (culture) phase with absence of steady SOX9 expression. Further building on this postulation, the failure to robustly express SOX9 may represent one of the bottlenecks to induce an efficient hormonal differentiation program in the organoids.

That the dense organoids display pituitary commitment (or at least 'memory') is further supported by the finding that they can give rise to hormonal cells, although so far only in vivo and at modest efficiency. Of note, although further expansion of $\mathrm{PRL}^{+}$cells developed in the grafts could be expected in the absence of negative hypothalamic feedback, the cells disappeared as part of an overall reduction of the grafts after the prolonged (6-week) stay in the ectopic subrenal location. It should also be noted that even xenografting of (human) pituitary tumors under the kidney capsule is not successful (Mertens et al. 2015), indicating that the kidney environment is not (yet) the optimal location for proper growth and maintenance of pituitary cells. In addition, hormonal cells in the pituitary are organized in networks (Le Tissier et al. 2012), and absence of these networks in the subrenal grafts because of the limited number of hormonal cells may also affect their survival.

Our organoid model also revealed aspects on pathways which may regulate pituitary stem cells, in particular WNT/LGR, and advanced LGR5 as a potential new marker of activated pituitary stem cells, likely responding to the increased RSPO1 levels in the gland upon damage (as found here). While LGR5+ cells represent stem cells in several epithelial tissues with fast turnover (such as intestine and stomach; Sato et al. 2009, Barker et al. 2010, Koo \& Clevers 2014), LGR5 expression in organs with slow turnover (such as liver and pancreas) only appears after tissue damage, with the emerging LGR5 ${ }^{+}$cells playing a role in the tissue's repair (Huch et al. $2013 a, b)$. A similar process seems to occur in the pituitary, similarly turning over at slow pace, with elevated LGR5 expression after damage (and in the corresponding cystic organoids). The importance of LGR5 as a new candidate marker of pituitary stem cell activation and the function of the LGR5+ cells represent appealing future research questions. Endogenous WNT signaling appears critical for cystic organoid formation and expansion since inhibiting the pathway strongly impedes these processes, thus suggesting the presence of an intrinsic WNT system in the organoid-forming (stem) cells. Taken together, the WNT/LGR5 pathway may be important in pituitary stem cell regulation as well as activation for regeneration, as also found in other organs (Clevers et al. 2014). In addition, we observed high expression of Prrx 1 and Prxx2 in the cystic organoids which led to the discovery of their upregulated expression in the gland upon damage. PRRX1 and PRRX2 have been described as putative pituitary stem cell markers (Vankelecom 2010, Higuchi et al. 2014, Cox et al. 2017). The majority of early-postnatal SOX2+ cells co-express PRRX1 but expression of PRRX1 decreases toward adulthood (Higuchi et al. 2014). Our observation of increased Prrx $1 / 2$ expression after damage may contribute to elucidating the pituitary (stem cell) activation and regeneration status after injury.

Finally, removal of nicotinamide, initially added to the organoid culture medium based on previous reports (Sato et al. 2011, Huch et al. 2013a,b), led to upregulation of $L h x 3$ expression in cystic organoids to levels even above the AP as a whole, as well as to an increase of hormone expression. LHX3 is indispensable for proper pituitary development and maturation (Zhu et al. 2007, Willems \& Vankelecom 2014). The pathway affected by nicotinamide may thus play a role in pituitary stem/progenitor cell specification and differentiation. At the concentrations used, nicotinamide acts as a histone deacetylase inhibitor by inhibiting the sirtuin enzyme family (Denu 2005). Sirtuins influence gene expression by, among others, epigenetic modifications such as histone deacetylation (Villalba \& Alcaín 2012). Inhibition of this sirtuin activity by nicotinamide may directly or indirectly repress $\operatorname{Lh} x 3$ expression. We observed differentiation of $\mathrm{ACTH}^{+}$and 
AGSU+ cells, although still infrequently, in the cystic organoids, especially when pre-cultured in the absence of nicotinamide. During pituitary embryogenesis AGSU and ACTH are the first hormones to appear (Zhu et al. 2007). Also in the ESC-derived pituitary-resembling 3D 'organoids' (Suga et al. 2011, Ozone et al. 2016) and in the iPSC-derived 2D cell cultures (Dincer et al. 2013, Zimmer et al. 2016), $\mathrm{ACTH}^{+}$cells were most efficiently derived. Differentiation to $\mathrm{ACTH}^{+}$cells appears to be the 'default' pathway in the pituitary, as also supported in transgenic mouse models in which cells adopt a corticotrope identity $\left(\mathrm{ACTH}^{+}\right.$cells) when development of other lineages is genetically compromised (Zhu et al. 2007). Of note, knockdown of $L h x 3$ resulted in a strong reduction of ACTH levels in the ESC-derived organoid cultures (Suga et al. 2011). Our results showing increased expression of $L h x 3$ and more clear ACTH (in the absence of nicotinamide) are in agreement with these findings. Finally, the presence of FGF8 in PitOM may also push differentiation to the corticotrope lineage as described before (Zhu et al. 2007, Zimmer et al. 2016). Why differentiation of $\mathrm{GH}^{+}$cells is not observed in vitro in the cystic organoid cultures whereas putatively occurring in vivo in the regenerating GHCre/iDTR pituitary is not clear yet but may have to do with the as yet absence in vitro of the needed paracrine or endocrine differentiation factor(s) which are present in the injured pituitary environment in vivo. In future research, our organoid model may be instrumental to uncover these factors that direct stem cells toward $\mathrm{GH}^{+}$cells.

In conclusion, we developed an organoid model for pituitary which may eventually lead to an important research tool to decipher pituitary stem cell biology and achieve deeper insight into their identity, regulation and activation. Additional scrutiny may reveal still undefined markers and identify potential stem cell (sub-) populations. Further optimization of the expandability and differentiation efficiency of the (cystic) organoids will allow to search for cellular and molecular pathways underlying pituitary regeneration to eventually identify potential regenerative paths and to in vitro model pituitary tissue as well as pituitary disease.

\section{Supplementary data}

This is linked to the online version of the paper at https://doi.org/10.1530/ JOE-18-0462.

\section{Declaration of interest}

The authors declare that there is no conflict of interest that could be perceived as prejudicing the impartiality of the research reported.

\section{Funding}

This work was supported by grants from the Fund for Scientific Research (FWO) - Flanders, Belgium (G.0674.14 and G0E1417N). B C, A V, C N and I V Z are supported by a PhD Fellowship from the FWO $(1141717 \mathrm{~N}, 11 \mathrm{~W} 9217 \mathrm{~N}$, $1 \mathrm{~S} 14218 \mathrm{~N}$ and $11 \mathrm{~F} 5419 \mathrm{~N}$, respectively), and $\mathrm{H} \mathrm{R}$ was supported by a PhD Fellowship from the IWT (Agency for Innovation by Science and Technology) - Flanders, Belgium (131557). M B is a PhD Fellow supported by a GOA grant from the Research Fund of the KU Leuven (GOA/14/012).

\section{Acknowledgements}

The authors thank Rob Van Rossom (Stem Cell Institute, KU Leuven) for help with FACS analysis and sorting, and the VIB Nucleomics Core (http:// www.nucleomics.be) and KU Leuven Genomics Core for expert assistance in RNA-seq analysis. They are also grateful to Yvonne Van Goethem for technical assistance. They thank Jos Laureys (Department of Chronic Diseases, Metabolism and Ageing, Clinical and Experimental Endocrinology, $\mathrm{KU}$ Leuven) for skilled assistance with in vivo transplantations. They are grateful to Dr Cynthia Andoniadou (King's College London, UK) for sharing RNAscope expertise. They thank InfraMouse (KU Leuven-VIB, Hercules type 3 grant) and the Cell Imaging Core (CIC; KU Leuven) for use of microscopes and histology equipment.

\section{References}

Andoniadou CL, Matsushima D, Mousavy Gharavy SN, Signore M, Mackintosh AI, Schaeffer M, Gaston-Massuet C, Mollard P, Jacques TS, Le Tissier P, et al. 2013 Sox2(+) stem/progenitor cells in the adult mouse pituitary support organ homeostasis and have tumor-inducing potential. Cell Stem Cell 13 433-445. (https://doi.org/10.1016/j. stem.2013.07.004)

Barker N, van Es JH, Kuipers J, Kujala P, van den Born M, Cozijnsen M, Haegebarth A, Korving J, Begthel H, Peters JP, et al. 2007 Identification of stem cells in small intestine and colon by marker gene Lgr5. Nature 449 1003-1007. (https://doi.org/10.1038/nature06196)

Barker N, Huch M, Kujala P, van de Wetering M, Snippert HJ, van Es JH, Sato T, Stange DE, Begthel H, van den Born M, et al. 2010 Lgr5(+ve) stem cells drive self-renewal in the stomach and build long-lived gastric units in vitro. Cell Stem Cell 6 25-36. (https://doi. org/10.1016/j.stem.2009.11.013)

Bilodeau S, Roussel-Gervais A \& Drouin J 2009 Distinct developmental roles of cell cycle inhibitors p57Kip2 and p27Kip1 distinguish pituitary progenitor cell cycle exit from cell cycle reentry of differentiated cells. Molecular and Cellular Biology 29 1895-1908. (https://doi.org/10.1128/MCB.01885-08)

Boretto M, Cox B, Noben M, Hendriks N, Fassbender A, Roose H, Amant F, Timmerman D, Tomassetti C, Vanhie A, et al. 2017 Development of organoids from mouse and human endometrium showing endometrial epithelium physiology and long-term expandability. Development 144 1775-1786. (https://doi.org/10.1242/ dev.148478)

Buch T, Heppner FL, Tertilt C, Heinen TJ, Kremer M, Wunderlich FT, Jung S \& Waisman A 2005 A Cre-inducible diphtheria toxin receptor mediates cell lineage ablation after toxin administration. Nature Methods 2 419-426. (https://doi.org/10.1038/nmeth762)

Buslei R, Hölsken A, Hofmann B, Kreutzer J, Siebzehnrubl F, Hans V, Oppel F, Buchfelder M, Fahlbusch R \& Blümcke I 2007 Nuclear betacatenin accumulation associates with epithelial morphogenesis in craniopharyngiomas. Acta Neuropathologica 113 585-590. (https://doi. org/10.1007/s00401-006-0184-3)

Candi E, Rufini A, Terrinoni A, Dinsdale D, Ranalli M, Paradisi A, De Laurenzi V, Spagnoli LG, Catani MV, Ramadan S, et al. 2006 https://joe.bioscientifica.com https://doi.org/10.1530/JOE-18-0462 (c) 2019 Society for Endocrinology Published by Bioscientifica Ltd. Printed in Great Britain 
Differential roles of p63 isoforms in epidermal development: selective genetic complementation in p63 null mice. Cell Death and Differentiation 13 1037-1047. (https://doi.org/10.1038/ sj.cdd.4401926)

Charles MA, Suh H, Hjalt TA, Drouin J, Camper SA \& Gage PJ 2005 PITX genes are required for cell survival and Lhx3 activation. Molecular Endocrinology 19 1893-1903. (https://doi.org/10.1210/me.2005-0052)

Chen J, Hersmus N, Van Duppen V, Caesens P, Denef C \& Vankelecom H 2005 The adult pituitary contains a cell population displaying stem/ progenitor cell and early embryonic characteristics. Endocrinology 146 3985-3998. (https://doi.org/10.1210/en.2005-0185)

Chen J, Gremeaux L, Fu Q, Liekens D, Van Laere S \& Vankelecom H 2009 Pituitary progenitor cells tracked down by side population dissection. Stem Cells 27 1182-1195. (https://doi.org/10.1002/stem.51)

Chen M, Kato T, Higuchi M, Yoshida S, Yako H, Kanno N \& Kato Y 2013 Coxsackievirus and adenovirus receptor-positive cells compose the putative stem/progenitor cell niches in the marginal cell layer and parenchyma of the rat anterior pituitary. Cell and Tissue Research 354 823-836. (https://doi.org/10.1007/s00441-013-1713-8)

Cheng Y, Xiang Y, Lin Y, Fu S, Jia W, Zhang G, Lv W, Mi S \& Zhao Q 2011 Retinoic acid and dexamethasone induce differentiation and maturation of somatotroph cells at different stages in vitro. Endocrine Journal 58 177-184. (https://doi.org/10.1507/endocrj.K10E-315)

Clevers H 2016 Modeling development and disease with organoids. Cell 165 1586-1597. (https://doi.org/10.1016/i.cell.2016.05.082)

Clevers H, Loh KM \& Nusse R 2014 Stem cell signaling. An integral program for tissue renewal and regeneration: Wnt signaling and stem cell control. Science 346 1248012. (https://doi.org/10.1126/ science.1248012)

Cox B, Roose H, Vennekens A \& Vankelecom H 2017 Pituitary stem cell regulation : who is pulling the strings? Journal of Endocrinology $\mathbf{2 3 4}$ R135-R158. (https://doi.org/10.1530/JOE-17-0083)

Denu JM 2005 Vitamin B3 and sirtuin function. Trends in Biochemical Sciences 30 479-483. (https://doi.org/10.1016/j.tibs.2005.07.004)

De Jaime-Soguero A, Aulicino F, Ertaylan G, Griego A, Cerrato A, Tallam A, Del Sol A, Cosma MP \& Lluis F 2017 Wnt/Tcf1 pathway restricts embryonic stem cell cycle through activation of the Ink4/Arf locus. PLoS Genetics 13 e1006682. (https://doi.org/10.1371/journal. pgen.1006682)

Dincer Z, Piao J, Niu L, Ganat Y, Kriks S, Zimmer B, Shi SH, Tabar V \& Studer L 2013 Specification of functional cranial placode derivatives from human pluripotent stem cells. Cell Reports 5 1387-1402 (https:// doi.org/10.1016/j.celrep.2013.10.048)

Ellis P, Fagan BM, Magness ST, Hutton S, Taranova O, Hayashi S, McMahon A, Rao M \& Pevny L 2004 SOX2, a persistent marker for multipotential neural stem cells derived from embryonic stem cells, the embryo or the adult. Developmental Neuroscience 26 148-165. (https://doi.org/10.1159/000082134)

Fauquier T, Rizzoti K, Dattani M, Lovell-Badge R \& Robinson IC 2008 SOX2-expressing progenitor cells generate all of the major cell types in the adult mouse pituitary gland. PNAS 105 2907-2912. (https:// doi.org/10.1073/pnas.0707886105)

Fu Q \& Vankelecom H 2012 Regenerative capacity of the adult pituitary: multiple mechanisms of lactotrope restoration after transgenic ablation. Stem Cells and Development 21 3245-3257. (https://doi. org/10.1089/scd.2012.0290)

Fu Q, Gremeaux L, Luque RM, Liekens D, Chen J, Buch T, Waisman A, Kineman R \& Vankelecom H 2012 The adult pituitary shows stem/ progenitor cell activation in response to injury and is capable of regeneration. Endocrinology 153 3224-3235. (https://doi.org/10.1210/ en.2012-1152)

Gage PJ \& Suh H 1999 The bicoid-related Pitx gene family in development. Mammalian Genome 10 197-200. (https://doi. org/10.1007/s003359900970)

Gao D, Vela I, Sboner A, Iaquinta PJ, Karthaus WR, Gopalan A, Dowling C, Wanjala JN, Undvall EA, Arora VK, et al. 2014 Organoid cultures derived from patients with advanced prostate cancer. Cell 159 176-187. (https://doi.org/10.1016/j.cell.2014.08.016)

Garcia-Lavandeira M, Quereda V, Flores I, Saez C, Diaz-Rodriguez E, Japon MA, Ryan AK, Blasco MA, Dieguez C, Malumbres M, et al. 2009 A GRFa2/Prop1/Stem (GPS) cell niche in the pituitary. PLOS ONE 4 e4815. (https://doi.org/10.1371/journal.pone.0004815)

Gaston-Massuet C, Andoniadou CL, Signore M, Jayakody SA, Charolidi N, Kyeyune R, Vernay B, Jacques TS, Taketo MM, Le Tissier P, et al. 2011 Increased Wingless (Wnt) signaling in pituitary progenitor/stem cells gives rise to pituitary tumors in mice and humans. PNAS $\mathbf{1 0 8}$ 11482-11487. (https://doi.org/10.1073/pnas.1101553108)

Goldsmith S, Lovell-Badge R \& Rizzoti K 2016 SOX2 is sequentially required for progenitor proliferation and lineage specification in the developing pituitary. Development 143 2376-2388. (https://doi org/10.1242/dev.137984)

Gregorieff A, Liu Y, Inanlou MR, Khomchuk Y \& Wrana JL 2015 Yapdependent reprogramming of Lgr5(+) stem cells drives intestinal regeneration and cancer. Nature 526 715-718. (https://doi. org/10.1038/nature15382)

Gremeaux L, Fu Q, Chen J \& Vankelecom H 2012 Activated phenotype of the pituitary stem/progenitor cell compartment during the earlypostnatal maturation phase of the gland. Stem Cells and Development 21 801-813. (https://doi.org/10.1089/scd.2011.0496)

Higuchi M, Yoshida S, Ueharu H, Chen M, Kato T \& Kato Y 2014 PRRX1 and PRRX2 distinctively participate in pituitary organogenesis and a cell-supply system. Cell and Tissue Research 357 323-335. (https://doi. org/10.1007/s00441-014-1861-5)

Huch M, Bonfanti P, Boj SF, Sato T, Loomans CL, van de Wetering M, Sojoodi M, Li VS, Schuijers J, Gracanin A, et al. 2013a Unlimited in vitro expansion of adult bi-potent pancreas progenitors through the Lgr5/R-spondin axis. EMBO Journal 32 2708-2721. (https://doi. org/10.1038/emboj.2013.204)

Huch M, Dorrell C, Boj SF, van Es JH, Li VS, van de Wetering M, Li VI, Hamer K, Sasaki N, Finegold MJ, et al. $2013 b$ In vitro expansion of single Lgr5+ liver stem cells induced by Wnt-driven regeneration. Nature 494 247-250. (https://doi.org/10.1038/nature11826)

Huch M, Gehart H, van Boxtel R, Hamer K, Blokzijl F, Verstegen MM, Ellis E, van Wenum M, Fuchs SA, de Ligt J, et al. 2015 Long-term culture of genome-stable bipotent stem cells from adult human liver. Cell 160 299-312. (https://doi.org/10.1016/j.cell.2014.11.050)

Hughes M \& Chuong CM 2003 A mouthful of epithelial-mesenchymal interactions. Journal of Investigative Dermatology 121 vii-viii. (https:// doi.org/10.1111/j.1523-1747.2003.12651.x)

Koo BK \& Clevers H 2014 Stem cells marked by the R-spondin receptor LGR5. Gastroenterology 147 289-302. (https://doi.org/10.1053/j. gastro.2014.05.007)

Lanctôt C, Lamolet B \& Drouin J 1997 The bicoid-related homeoprotein Ptx1 defines the most anterior domain of the embryo and differentiates posterior from anterior lateral mesoderm. Development 124 2807-2817.

Lay K, Kume T \& Fuchs E 2016 FOXC1 maintains the hair follicle stem cell niche and governs stem cell quiescence to preserve long-term tissue-regenerating potential. PNAS 113 E1506-E1515. (https://doi. org/10.1073/pnas.1601569113)

Le Tissier PR, Hodson DJ, Lafont C, Fontanaud P, Schaeffer M \& Mollard P 2012 Anterior pituitary cell networks. Frontiers in Neuroendocrinology 33 252-266. (https://doi.org/10.1016/j.yfrne.2012.08.002)

Luque RM, Amargo G, Ishii S, Lobe C, Franks R, Kiyokawa H \& Kineman RD 2007 Reporter expression, induced by a growth hormone promoter-driven Cre recombinase (RGHp-Cre) transgene, questions the developmental relationship between somatotropes and lactotropes in the adult mouse pituitary gland. Endocrinology 148 1946-1953. (https://doi.org/10.1210/en.2006-1542)

Luque RM, Lin Q, Córdoba-Chacón J, Subbaiah PV, Buch T, Waisman A, Vankelecom H \& Kineman RD 2011 Metabolic impact of adult-onset, isolated, growth hormone deficiency (AOiGHD) due to destruction of https://joe.bioscientifica.com

https://doi.org/10.1530/JOE-18-0462 (c) 2019 Society for Endocrinology Published by Bioscientifica Ltd. Printed in Great Britain 
pituitary somatotropes. PLoS ONE 6 e15767. (https://doi.org/10.1371/ journal.pone.0015767)

Mertens F, Gremeaux L, Chen J, Fu Q, Willems C, Roose H, Govaere O, Roskams T, Cristina C, Becú-Villalobos D, et al. 2015 Pituitary tumors contain a side population with tumor stem cell-associated characteristics. Endocrine-Related Cancer 22 481-504. (https://doi. org/10.1530/ERC-14-0546)

Moll R, Divo M \& Langbein L 2008 The human keratins: biology and pathology. Histochemistry and Cell Biology 129 705-733. (https://doi. org/10.1007/s00418-008-0435-6)

Ozone C, Suga H, Eiraku M, Kadoshima T, Yonemura S, Takata N, Oiso Y, Tsuji T \& Sasai Y 2016 Functional anterior pituitary generated in selforganizing culture of human embryonic stem cells. Nature 710351. (https://doi.org/10.1038/ncomms10351)

Rizzoti K, Akiyama H \& Lovell-Badge R 2013 Mobilized adult pituitary stem cells contribute to endocrine regeneration in response to physiological demand. Cell Stem Cell 13 419-432. (https://doi. org/10.1016/j.stem.2013.07.006)

Roose H, Cox B, Boretto M, Gysemans C, Vennekens A \& Vankelecom H 2017 Major depletion of SOX2+ stem cells in the adult pituitary is not restored which does not affect hormonal cell homeostasis and remodelling. Scientific Reports 7 1-11. (https://doi.org/10.1038/ s41598-016-0028-x)

Sato T, Vries RG, Snippert HJ, van de Wetering M, Barker N, Stange DE, van Es JH, Abo A, Kujala P, Peters PJ, et al. 2009 Single Lgr5 stem cells build crypt-villus structures in vitro without a mesenchymal niche. Nature 459 262-265. (https://doi. org/10.1038/nature07935)

Sato T, Stange DE, Ferrante M, Vries RG, Van Es JH, Van den Brink S, Van Houdt WJ, Pronk A, Van Gorp J, Siersema PD, et al. 2011 Long-term expansion of epithelial organoids from human colon, adenoma, adenocarcinoma, and Barrett's epithelium. Gastroenterology 141 1762-1772. (https://doi.org/10.1053/j.gastro.2011.07.050)

Sierra A, Martín-Suárez S, Valcárcel-Martín R, Pascual-Brazo J, Aelvoet SA, Abiega O, Deudero JJ, Brewster AL, Bernales I, Anderson AE, et al. 2015 Neuronal hyperactivity accelerates depletion of neural stem cells and impairs hippocampal neurogenesis. Cell Stem Cell 16 488-503. (https://doi.org/10.1016/j.stem.2015.04.003)

Spandidos A, Wang X, Wang H \& Seed B 2010 PrimerBank: a resource of human and mouse PCR primer pairs for gene expression detection and quantification. Nucleic Acids Research 38 D792-D799. (https://doi. org/10.1093/nar/gkp1005)

Suga H, Kadoshima T, Minaguchi M, Ohgushi M, Soen M, Nakano T, Takata N, Wataya T, Muguruma K, Miyoshi H, et al. 2011
Self-formation of functional adenohypophysis in threedimensional culture. Nature $\mathbf{4 8 0}$ 57-62 (https://doi.org/10.1038/ nature10637)

Tateyama H, Tada T, Okabe M, Takahashi E \& Eimoto T 2001 Different keratin profiles in craniopharyngioma subtypes and ameloblastomas. Pathology: Research and Practice 197 735-742. (https://doi. org/10.1078/0344-0338-00152)

Vankelecom H 2010 Pituitary stem/progenitor cells: embryonic players in the adult gland? European Journal of Neuroscience 32 2063-2081. (https://doi.org/10.1111/j.1460-9568.2010.07523.x)

Vankelecom H 2012 Pituitary stem cells drop their mask. Current Stem Cell Research and Therapy 7 36-71. (https://doi. org/10.2174/157488812798483467)

Van Keymeulen A, Rocha AS, Ousset M, Beck B, Bouvencourt G, Rock J, Sharma N, Dekoninck S \& Blanpain C 2011 Distinct stem cells contribute to mammary gland development and maintenance. Nature 479 189-193. (https://doi.org/10.1038/nature10573)

Villalba JM \& Alcaín FJ 2012 Sirtuin activators and inhibitors. BioFactors 38 349-359. (https://doi.org/10.1002/biof.1032)

Willems C \& Vankelecom H 2014 Pituitary cell differentiation from stem cells and other cells: toward restorative therapy for hypopituitarism? Regenerative Medicine 9 513-534. (https://doi. org $/ 10.2217 /$ rme.14.19)

Willems C, Fu Q, Roose H, Mertens F, Cox B, Chen J \& Vankelecom H 2016 Regeneration in the pituitary after cell-ablation injury: timerelated aspects and molecular analysis. Endocrinology 157 705-721. (https://doi.org/10.1210/en.2015-1741)

Xin W, Rubin MA \& McKeever PE 2002 Differential expression of cytokeratins 8 and 20 distinguishes craniopharyngioma from Rathke cleft cyst. Archives of Pathology \& Laboratory Medicine 126 1174-1178. (https://doi.org/10.1043/0003-9985(2002)126<1174:DEOC $\mathrm{AD}>2.0 . \mathrm{CO} ; 2)$

Yu WY, Slack JM \& Tosh D 2005 Conversion of columnar to stratified squamous epithelium in the developing mouse oesophagus. Developmental Biology 284 157-170. (https://doi.org/10.1016/j. ydbio.2005.04.042)

Zhu X, Gleiberman AS \& Rosenfeld MG 2007 Molecular physiology of pituitary development: signaling and transcriptional networks. Physiological Reviews 87 933-963. (https://doi.org/10.1152/ physrev.00006.2006)

Zimmer B, Piao J, Ramnarine K, Tomishima MJ, Tabar V \& Studer L 2016 Derivation of diverse hormone-releasing pituitary cells from human pluripotent stem cells. Stem Cell Reports 6 858-872. (https://doi. org/10.1016/j.stemcr.2016.05.005)

Received in final form 17 November 2018

Accepted 23 November 2018

Accepted Preprint published online 26 November 2018 (c) 2019 Society for Endocrinology Published by Bioscientifica Ltd. Printed in Great Britain 